\title{
BLIND BACKSCATTERING EXPERIMENTAL DATA COLLECTED IN THE FIELD AND AN APPROXIMATELY GLOBALLY CONVERGENT INVERSE ALGORITHM
}

\author{
ANDREY V. KUZHUGET*॰ ${ }^{*}$ LARISA BEILINA**o , MICHAEL V. KLIBANOV ${ }^{\ddagger \circ}$, ANDERS SULLIVAN ${ }^{\dagger}$, LAM \\ NGUYEN $\diamond \bullet$, AND MICHAEL A. FIDDY $\triangle \circ$
}

\begin{abstract}
.
An approximately globally convergent numerical method for a 1-D Coefficient Inverse Problem for a hyperbolic PDE is applied to image dielectric constants of targets from blind experimental data. The data were collected in the field by the Forward Looking Radar of the US Army Research Laboratory. A posteriori analysis has revealed that computed and tabulated values of dielectric constants are in a good agreement. Convergence analysis is presented.
\end{abstract}

1. Introduction. In this paper we test the 1-D version [31] of the numerical method of recent publications $[5,6,7,8,9,10,11,12,27,28,32,48]$ for the case when the time resolved backscattering electric signal is measured experimentally in the field. Measurements were conducted by the Forward Looking Radar built in US Army Research Laboratory (ARL). All kinds of clutter were present at the site of data collection. The data are severely limited. The goal of this radar is to detect and possibly identify shallow explosive-like targets. Prior to this effort, the focus of the ARL team was on the image processing rather than on the target detection and identification [37]. The current data processing procedure of ARL delivers only the energy information. The algorithm of this paper computes values of dielectric constants of targets using those data. These values represent a new, surprising and quite useful dimension of information for the ARL team. A hope is that these values might be helpful in the target detection and identification process.

The UNCC/ChalmersGU team has worked only with the most challenging case of blind experimental data. "Blind" means that first computations were made by the UNCC/ChalmersGU team without any knowledge of correct answers. Next, computational results were sent to the ARL team. The ARL team has compared a posteriori those results with the reality and then revealed correct answers to the UNCC/ChalmersGU team. The performance of the algorithm of above cited publications for transmitted blind experimental data was presented in [27], see Tables 5 and 6 there. Images of [27] were refined in the follow up publication [9] using the adaptivity technique of [3, 4].

In the above cited works a new numerical method was developed for some Multidimensional Coefficient Inverse Problems (MCIPs) for a hyperbolic PDE with single measurement data. "Single measurement" means that either only a single position of the point source or only a single direction of the incident plane wave is considered. Because of many dangers on the battlefield, the single measurement arrangement is the most suitable one for military applications. There were two goals of those publications:

Goal 1. To develop such a numerical method, which would have a rigorous guarantee obtaining a good approximation for the exact solution of a CIP without using an advanced knowledge of neither a small neighborhood of that solution nor of the background medium in the domain of interest.

Goal 2. This method should demonstrate a good performance on both computationally simulated and experimental data.

It is well known that it is enormously challenging to achieve both Goals 1,2 simultaneously. Three substantial obstacles are combined here: the minimal information content due to the single source only, nonlinearity and ill-posedness. Therefore, it was inevitable in the above cited publications to introduce

\footnotetext{
${ }^{*}$ Morgan Stanley \& Co. Incorporated, 1585 Broadway, New York, NY 10036, USA (formely with University of North Carolina at Charlotte)

** Department of Mathematical Sciences, Chalmers University of Technology and Gothenburg University, SE-42196 Gothenburg, Sweden, email: larisa@chalmers.se

$¥$ Corresponding author, Department of Mathematics and Statistics, University of North Carolina at Charlotte, Charlotte, NC 28223, USA, email: mklibanv@uncc.edu

† US Army Research Laboratory, 2800 Powder Mill Road Adelphy, MD 20783-1197, USA,email: anders.j.sullivan.civ@mail.mil

• US Army Research Laboratory, 2800 Powder Mill Road Adelphy, MD 20783-1197, USA,email: lam.h.nguyen2.civ@mail.mil

$\triangle$ Optoelectronics Center, University of North Carolina at Charlotte, Charlotte, NC 28223, USA, email: mafiddy@uncc.edu

○ UNCC/ChalmersGU team, • ARL team
} 
some natural approximations. Although those approximations cannot be rigorously justified sometimes, still they have resulted in the simultaneous achievement of both goals, see more details in sections 4,6. The numerical method of $[5,6,7,8,9,10,11,12,27,28,31,32,48]$ uses the structure of the underlying PDE operator rather than a least squares functional. The above thoughts are reflected in the following statement of the review paper [12] "The key philosophical focus of our review is the above point about natural assumptions/approximations which make the technique numerically efficient."

Because of the measurement scheme of the Forward Looking Radar, the UNCC/ChalmersGU team had only one time resolved curve for each target. This curve was the voltage averaged over some readings (section 7). Only one component of the electric field was generated and measured in the backscattering regime. The reality is 3-D, and the electric field propagation is governed by the full Maxwell's system. However, the above data structure has left us no choice but to model the process by a 1-D CIP using only one hyperbolic PDE.

The main challenge of working with these data was a huge misfit between them and computationally simulated data, e.g. compare Figure 7.3-b) with Figures 7.4-b), d), f). Namely, the experimentally measured curves are highly oscillatory, unlike computed ones. Therefore, to make the experimental data suitable for the inversion, we apply a new data pre-processing procedure, which is a crucial step. This procedure was unbiased, since we have worked with the blind data only. See [9, 27] for another data pre-processing procedure for the case of transmitted data. Both these procedures are based on the intuition only. The only justification for both is the accuracy of obtained results.

There are some classical numerical methods of solving 1-D CIPs for hyperbolic equations, see, e.g. $[19,25,36]$ and references cited therein. However, because of many uncertainties in our experimental data, including the above misfit and the 3-D reality versus the 1-D mathematical model (also, see subsection 7.2), it is yet unclear how those methods would perform for our data. This question deserves a separate research effort.

There are also MCIPs with multiple measurement data. "Multiple measurements" means that the data are generated either by the point source running along a manifold, or by the incident plane wave, whose direction varies within a certain cone. These MCIPs have applications in medical imaging. We refer to $[1,13,16,17,21,24,38,39,40,41]$ for some non-local reconstruction techniques for these MCIPs. An analog of the technique of [5] for the case of a 2-D CIP for an elliptic PDE with the source running along a straight line was developed $[29,42,43]$. Validation of the latter for the case of experimental data with the application to stroke detection in brains of small animals was done in [44]. We point out that one of the main keys to the success of numerical results of $[1,16,17]$ for the reconstruction algorithms of Novikov $[38,39,40,41]$ is the use of approximate mathematical models. The same can be said about $[29,42,43,44]$. This concept is similar with the one above.

In section 2 we pose a CIP for a 1-D wave-like PDE. In section 3 we study some properties of the Laplace transform of the solution of the forward problem. In section 4 we discuss the concept of the approximate global convergence property. In section 5 we present our numerical method. Convergence analysis can be found in section 6 . In section 7 we describe the experimental setup, main uncertainties in experimental data and the data pre-processing procedure. Imaging results are presented in section 8 .

2. Statements of Forward and Inverse Problems. Let the function $\varepsilon_{r}(x), x \in \mathbb{R}$ be the dielectric constant, spatially distributed on the real line. Let the number $d>1$. We assume below that

$$
\begin{gathered}
\varepsilon_{r}(x) \in[1, d), \varepsilon_{r} \in C^{1}(\mathbb{R}), \\
\varepsilon_{r}(x)=1, x \notin(0,1) .
\end{gathered}
$$

Thus, the interval $(0,1)$ is our domain of interest in the inverse problem. The forward problem is

$$
\begin{gathered}
\varepsilon_{r}(x) u_{t t}=u_{x x}, x \in \mathbb{R}, t \in(0, \infty), \\
u(x, 0)=0, u_{t}(x, 0)=\delta\left(x-x_{0}\right),
\end{gathered}
$$




$$
x_{0}=\text { const. }<0 \text {. }
$$

Coefficient Inverse Problem. Determine the coefficient $\varepsilon_{r}(x)$, assuming that the following function $g(t)$ is known

$$
u(0, t)=g(t), t \in(0, \infty) .
$$

The function $g(t)$ models the backscattering data measured by the Forward Looking Radar. The condition $t \in(0, \infty)$ in $(2.6)$ is not a restrictive one from the numerical standpoint. Indeed, we use the Laplace transform (2.7) to solve this CIP. Since the kernel $e^{-s t}$ of this transform decays rapidly with respect to $t$, then in the actuality a finite time interval is sufficient. In addition, the data resulting from the pre-processing procedure have a finite support in $(0, \infty)$. Since this is the 1 -D case, uniqueness theorems for this CIP are well known, see, e.g. [36] and references cited therein.

Consider the Laplace transform

$$
w(x, s)=\int_{0}^{\infty} u(x, t) e^{-s t} d t:=\mathcal{L} u, s \geq \underline{s}=\text { const. }>0,
$$

where the number $\underline{s}=\underline{s}\left(\varepsilon_{r}\right)$ is sufficiently large. We call the parameter s pseudo frequency. Hence,

$$
\begin{gathered}
w_{x x}-s^{2} \varepsilon_{r}(x) w=-\delta\left(x-x_{0}\right), x \in \mathbb{R}, \\
\lim _{|x| \rightarrow \infty} w(x, s)=0,
\end{gathered}
$$

see Theorem 3.1 for the proof of (2.9). By (2.6)

$$
w(0, s)=\varphi(s):=\mathcal{L} g, s \geq \underline{s} .
$$

We also need to know the derivative $w_{x}(0, s)$,

$$
w_{x}(0, s)=\rho(s), s \geq \underline{s} .
$$

Consider the fundamental solution $w_{0}(x, s)$ of the problem $(2.8),(2.9)$ for $\varepsilon_{r}(x) \equiv 1$. Then

$$
w_{0}(x, s)=(2 s)^{-1} \exp \left(-s\left|x-x_{0}\right|\right) \text {. }
$$

Denote $\widehat{w}(x, s)=w(x, s)-w_{0}(x, s)$. Using (2.8)-(2.10), we obtain

$$
\begin{gathered}
\widehat{w}_{x x}-s^{2} \varepsilon_{r}(x) \widehat{w}=s^{2}\left(\varepsilon_{r}(x)-1\right) w_{0}, x \in \mathbb{R}, \\
\lim _{|x| \rightarrow \infty} \widehat{w}(x, s)=0, \\
\widehat{w}(0, s)=\widehat{\varphi}(s):=\varphi(s)-(2 s)^{-1} \exp \left(-s\left|x_{0}\right|\right) .
\end{gathered}
$$

Also, (2.2) and (2.12)-(2.14) imply for $x<0$

$$
\begin{gathered}
\widehat{w}_{x x}-s^{2} \widehat{w}=0, x<0, \\
\lim _{x \rightarrow-\infty} \widehat{w}(x, s)=0, \\
\widehat{w}(0, s)=\widehat{\varphi}(s) .
\end{gathered}
$$

It follows from (2.15)-(2.17) that $\widehat{w}(x, s)=\widehat{\varphi}(s) e^{s x}, x<0$. Hence, $\widehat{w}_{x}(0, s)=s \widehat{\varphi}(s)=s(\varphi(s)-$ $\left.(2 s)^{-1} \exp \left(-s\left|x_{0}\right|\right)\right)$ and thus since $x_{0}<0$, we have

$$
w_{x}(0, s):=\rho(s)=s \varphi(s)-\exp \left(s x_{0}\right) .
$$


3. Some properties of the function $w(x, s)$. Below $C^{\alpha}, \alpha \in(0,1)$ are Hölder spaces. In this section we establish some properties of the function $w=\mathcal{L} u$, which we need for the convergence analysis. Let $f(t), t>0$ be a piecewise continuous function such that $|f(t)| \leq C e^{a t}, t>1$, where $C=C(f), a=a(f)=$ const. $>0$. Consider two types of the Laplace transform,

$$
\begin{gathered}
\mathcal{L}_{1}(f)(t)=\frac{1}{2 \sqrt{\pi} t^{3 / 2}} \int_{0}^{\infty} \tau \exp \left(-\frac{\tau^{2}}{4 t}\right) f(\tau) d \tau, \\
\mathcal{L}_{2}(f)(s)=\int_{0}^{\infty} f(t) e^{-s^{2} t} d t, s>\sqrt{a(f)} .
\end{gathered}
$$

Lemma 3.1. $\mathcal{L}_{2}\left[\mathcal{L}_{1}(f)\right](s)=\mathcal{L}(f)(s)$ for $s>a(f)$, where the operator $\mathcal{L}$ was defined in $(2.7)$.

Proof. Using the formula (28) of section 4.5 of the book [2], we obtain

$$
\int_{0}^{\infty}\left[\frac{1}{2 \sqrt{\pi} t^{3 / 2}} \tau \exp \left(-\frac{\tau^{2}}{4 t}\right)\right] e^{-s^{2} t} d t=e^{-s t}, \forall s>0 .
$$

Theorem 3.1. Consider the function $\varepsilon_{r}(x) \in C^{\alpha}(\mathbb{R})$ satisfying the rest of conditions (2.1), (2.2). Let (2.5) holds. Then for any number $s>0$ there exists unique solution $p(x, s) \in C^{2+\alpha}\left(\left|x-x_{0}\right| \geq \beta\right) \cap$ $C(\mathbb{R}), \forall \beta>0$ of the following problem

$$
\begin{gathered}
p_{x x}-s^{2} \varepsilon_{r}(x) p=-\delta\left(x-x_{0}\right), x \in \mathbb{R}, \\
\lim _{|x| \rightarrow \infty} p(x, s)=0 .
\end{gathered}
$$

Also,

$$
0<p(x, s) \leq w_{0}(x, s), \forall x \in \mathbb{R} .
$$

In addition, let $\left|x-x_{0}\right| \geq \beta=$ const. $>0$. Then there exists a sufficiently large number $\widehat{s}=\widehat{s}(d, \beta)$ such that

$$
p(x, s)>w_{d}(x, s), \forall x \in\left[x_{0}+\beta, \infty\right), \forall s \geq \widehat{s},
$$

where $w_{d}(x, s)$ is the fundamental solution of equation (2.8) for the case $\varepsilon_{r}(x) \equiv d$,

$$
w_{d}(x, s)=(2 s)^{-1} \exp \left(-s \sqrt{d}\left|x-x_{0}\right|\right) .
$$

Finally, there exists a sufficiently large number $\underline{s}=\underline{s}\left(\varepsilon_{r}\right)>0$ such that

$$
w(x, s):=\mathcal{L}(u)(x, s)=p(x, s), \forall s \geq \underline{s}\left(\varepsilon_{r}\right), \forall x \in \mathbb{R} .
$$

Thus, (3.2) implies that (2.9) holds for $s \geq \underline{s}=\underline{s}\left(\varepsilon_{r}\right)$.

Proof. Let the function $v(x, t)$ be the solution of the following Cauchy problem

$$
\begin{gathered}
\varepsilon_{r}(x) v_{t}=v_{x x},(x, t) \in \mathbb{R} \times(0, \infty), \\
v(x, 0)=\delta\left(x-x_{0}\right) .
\end{gathered}
$$


Let the function $v_{0}(x, t)$ be the solution of the problem $(3.7),(3.8)$ for the case $\varepsilon_{r}(x) \equiv 1$,

$$
v_{0}(x, t)=\frac{1}{2 \sqrt{\pi t}} \exp \left[-\frac{\left(x-x_{0}\right)^{2}}{4 t}\right] .
$$

Let $\widehat{v}(x, t)=v(x, t)-v_{0}(x, t)$. Then

$$
\widehat{v}_{x x}-\varepsilon_{r}(x) \widehat{v}_{t}=\left(\varepsilon_{r}(x)-1\right) v_{0 t}, \widehat{v}(x, 0)=0 .
$$

Detailed estimates of the fundamental solution of a general parabolic equation in chapter 4 of the book [33] imply that $\widehat{v} \in C^{2+\alpha, 1+\alpha / 2}(\mathbb{R} \times[0, T]), \forall T>0$. Denote

$$
\widetilde{v}(x, t)=\int_{0}^{t} \widehat{v}(x, \tau) d \tau=\int_{0}^{t}\left(v-v_{0}\right)(x, \tau) d \tau .
$$

By $(3.9)$

$$
\widetilde{v}_{x x}-\varepsilon_{r}(x) \widetilde{v}_{t}=\left(\varepsilon_{r}(x)-1\right) v_{0}, \widetilde{v}(x, 0)=0 .
$$

By (2.2), (2.5) $\varepsilon_{r}(x)-1=0$ in a neighborhood of the source point $\left\{x_{0}\right\}$. Hence, applying (2.2), we obtain $\left(\varepsilon_{r}(x)-1\right) v_{0}(x, t) \geq 0$ in $\mathbb{R} \times[0, \infty)$. Hence, applying the maximum principle of Theorem 1 of chapter 2 of the book [23] to (3.11) we obtain

$$
\widetilde{v}(x, t) \leq 0 \text { in } \mathbb{R} \times[0, \infty)
$$

On the other hand, Theorem 11 of chapter 2 of [23] ensures that the fundamental solution of the parabolic equation is positive for $t>0$. Hence,

$$
v(x, t)>0, t>0
$$

Hence, (3.10), (3.12) and (3.13) imply that

$$
0<\int_{0}^{t} v(x, \tau) d \tau \leq \int_{0}^{t} v_{0}(x, \tau) d \tau .
$$

Using Fubini theorem, formula (27) of section 4.5 of the book [2] and (2.11), we obtain

$$
\mathcal{L}_{2}\left(\int_{0}^{t} v_{0}(x, \tau) d \tau\right)=\frac{1}{s^{2}} \mathcal{L}_{2}\left(v_{0}\right)=\frac{w_{0}(x, s)}{s^{2}} .
$$

Hence, using (3.13), (3.14) and Fubini theorem, we obtain

$$
\mathcal{L}_{2}\left(\int_{0}^{t} v(x, \tau) d \tau\right)=\frac{1}{s^{2}} \mathcal{L}_{2}(v)(x, s):=\frac{y(x, s)}{s^{2}} \leq \frac{w_{0}(x, s)}{s^{2}},
$$

By (2.11) and (3.16)

$$
\lim _{|x| \rightarrow \infty} y(x, s)=0
$$


Next, by (3.10) and (3.11) $\widetilde{v}_{x x}=\varepsilon_{r}(x) v-v_{0}$. Hence, $\left|\widetilde{v}_{x x}\right| \leq d v+v_{0}$. Therefore,

$$
\int_{0}^{\infty}\left|\widetilde{v}_{x x}(x, t)\right| e^{-s^{2} t} d t<\infty .
$$

Hence,

$$
\partial_{x}^{2} \int_{0}^{\infty} \widetilde{v}(x, t) e^{-s^{2} t} d t=\int_{0}^{\infty} \widetilde{v}_{x x}(x, t) e^{-s^{2} t} d t=\varepsilon_{r}(x) y-w_{0} .
$$

On the other hand,

$$
\begin{gathered}
\int_{0}^{\infty} \widetilde{v}_{x x}(x, t) e^{-s^{2} t} d t=\int_{0}^{\infty}\left(\int_{0}^{t} v_{x x}(x, \tau) d \tau\right) e^{-s^{2} t} d t-\int_{0}^{\infty}\left(\int_{0}^{t} v_{0 x x}(x, \tau) d \tau\right) e^{-s^{2} t} d t \\
=s^{-2}\left(y_{x x}-w_{0 x x}\right) .
\end{gathered}
$$

Comparing this with (3.18) and keeping in mind that $w_{0 x x}-s^{2} w_{0}=-\delta\left(x-x_{0}\right)$, we obtain

$$
y_{x x}-s^{2} \varepsilon_{r}(x) y=-\delta\left(x-x_{0}\right) .
$$

It follows from(3.19) that the function $y(x, s)=\mathcal{L}_{2}(v)(x, s)$ satisfies conditions (3.1), (3.2). It follows from the above that $y(x, s) \in C^{2+\alpha}\left(\left|x-x_{0}\right| \geq \beta\right) \cap C(\mathbb{R}), \forall \beta, s>0$. Uniqueness of the solution of the problem (3.1), (3.2) for this class of functions can be easily proven in the standard way using the maximum principle for elliptic equations and (3.2). Hence, the problem (3.1), (3.2) has the unique solution $p(x, s):=y(x, s) \in$ $C^{2+\alpha}\left(\left|x-x_{0}\right| \geq \beta\right) \cap C(\mathbb{R}), \forall \beta, s>0$. The right estimate (3.3) follows from (3.16), and the left estimate follows from (3.16). Lemma 3.1 implies (3.6).

We now prove (3.4). We obtain from (2.2), (3.1) and (3.2)

$$
p(x, s)=\frac{\exp \left(-s\left|x-x_{0}\right|\right)}{2 s}-\frac{s}{2} \int_{0}^{1} \exp (-s|x-\xi|)\left(\varepsilon_{r}(\xi)-1\right) p(\xi, s) d \xi .
$$

Hence, by (2.1) and the right inequality (3.3)

$$
p(x, s) \geq \frac{\exp \left(-s\left|x-x_{0}\right|\right)}{2 s}-\frac{(d-1)}{4} \int_{0}^{1} \exp (-s|x-\xi|) \exp \left(-s\left|\xi-x_{0}\right|\right) d \xi .
$$

Assume now that $x \in\left(x_{0}, 0\right)$. Then

$$
\begin{aligned}
p(x, s) & \geq \frac{\exp \left[-s\left(x-x_{0}\right)\right]}{2 s}-\frac{(d-1) \exp \left[s\left(x+x_{0}\right)\right]}{4} \int_{0}^{1} e^{-2 s \xi} d \xi \\
& =\frac{\exp \left[-s\left(x-x_{0}\right)\right]}{2 s}\left[1-\frac{(d-1)\left(1-e^{-2 s}\right) e^{2 s x}}{4}\right] .
\end{aligned}
$$

Therefore

$$
\frac{p(x, s)}{w_{d}(x, s)} \geq \exp \left[s(\sqrt{d}-1)\left(x-x_{0}\right)\right]\left[{ }_{6}-\frac{(d-1)\left(1-e^{-2 s}\right) e^{2 s x}}{4}\right], \forall x \in\left(x_{0}, 0\right) .
$$


Hence, if $\beta=$ const. $\in\left(0,\left|x_{0}\right|\right)$, then there exists a number $\widehat{s}=\widehat{s}(d, \beta)$ such that

$$
\frac{p(x, s)}{w_{d}(x, s)}>1, \forall s \geq \widehat{s}=\widehat{s}(d, \beta), \forall x \in
$$

Consider now the difference $\widetilde{p}(x, s)=p(x, s)-w_{d}(x, s)$. Then

$$
\begin{gathered}
\widetilde{p}_{x x}-s^{2} \varepsilon_{r}(x) \tilde{p}=s^{2}\left(\varepsilon_{r}(x)-d\right) w_{d}<0, x \in\left[x_{0}+\beta, \infty\right), \\
\lim _{x \rightarrow \infty} \tilde{p}(x, s)=0 .
\end{gathered}
$$

Let $a>1$ be an arbitrary number. Consider the function $\widetilde{p}(x, s)$ on the interval $\left[x_{0}+\beta, a\right]$ for $s \geq \widehat{s}(d, \beta)$. Then the maximum principle for elliptic equations, (3.21) and (3.22) imply that the negative minimum of $\widetilde{p}(x, s)$ on this interval can be achieved only at $x=a$. Setting $a \rightarrow \infty$ and using (3.23), we obtain that $\widetilde{p}(x, s) \geq 0$ for $x \in\left[x_{0}+\beta, \infty\right), s \geq \widehat{s}(d, \beta)$.

Assume now that there exists a point $\bar{x} \in\left[x_{0}+\beta, \infty\right)$ and a number $s \geq \widehat{s}(d, \beta)$ such that $\widetilde{p}(\bar{x}, s)=0$. Since $\widetilde{p}(x, s) \geq 0$ for $x \in\left[x_{0}+\beta, \infty\right)$, then

$$
\widetilde{p}(\bar{x}, s)=0=\min _{\left[x_{0}+\beta, \infty\right)} \widetilde{p}(x, s) .
$$

Hence, $\widetilde{p}_{x x}(\bar{x}, s) \geq 0$. However, this and (3.24) contradict to the inequality in (3.22).

Corollary 3.1. Let the function $\varepsilon_{r}(x) \in C^{\alpha}(\mathbb{R})$, satisfies the rest of conditions (2.1), (2.2). For every $s>0$ and for every $x \in \mathbb{R}$ the integral of the Laplace transform $\mathcal{L}_{2}(v(x, t))$ converges absolutely.

Proof. See (3.14), (3.15) and (3.16).

Remark 3.1. By (3.6) the function $\varphi(s)$ in (2.10) is defined only for $s \geq \underline{s}\left(\varepsilon_{r}\right)$. However, using Lemma 3.1 and Corollary 3.1, we set below $\varphi(s):=\mathcal{L}_{2}\left[\mathcal{L}_{1}(g)\right](s), \forall s>0$, where the function $g(t)$ is defined in (2.6).

Theorem 3.2. Let the function $\varepsilon_{r}(x) \in C^{\alpha}(\mathbb{R})$, satisfies the rest of conditions (2.1), (2.2) and (2.5) holds. Let the number $\bar{s} \geq \widehat{s}\left(\left|x_{0}\right|, d\right)$, where the number $\widehat{s}$ was defined in Theorem 3.1. Let the function $p\left(x, \bar{s} ; \varepsilon_{r}\right):=p(x, \bar{s}) \in C^{2+\alpha}\left(\left|x-x_{0}\right| \geq \beta\right) \cap C(\mathbb{R}), \forall \beta>0$ be the solution of the problem (3.1), (3.2). Denote

$$
f_{k}\left(x, \bar{s} ; \varepsilon_{r}\right)=\bar{s}^{-2} \partial_{x}^{k}[\ln p(x, \bar{s})], k=1,2 .
$$

Then there exists a constant $B=B\left(x_{0}, d, \bar{s}\right)>1$ such that for all such functions $\varepsilon_{r}$

$$
\left\|f_{k}\left(x, \bar{s} ; \varepsilon_{r}\right)\right\|_{C[0,1]} \leq B, k=1,2 .
$$

Also, for any two functions $\varepsilon_{r}^{(1)}, \varepsilon_{r}^{(2)}$ satisfying conditions of this theorem

$$
\left\|f_{k}\left(x, \bar{s} ; \varepsilon_{r}^{(1)}\right)-f_{k}\left(x, \bar{s} ; \varepsilon_{r}^{(2)}\right)\right\|_{L_{2}(0,1)} \leq B\left\|\varepsilon_{r}^{(1)}-\varepsilon_{r}^{(2)}\right\|_{L_{2}(0,1)}, k=1,2 .
$$

Proof. In this proof $B=B\left(x_{0}, d, \bar{s}\right)>1$ denotes different constants depending on listed parameters. We have

$$
f_{1}=\frac{p_{x}}{\bar{s}^{2} p}, f_{2}=\frac{p_{x x}}{\bar{s}^{2} p}-\frac{p_{x}^{2}}{\bar{s}^{2} p^{2}} .
$$

Hence, by (3.4)

$$
\left|f_{1}\left(x, \bar{s} ; \varepsilon_{r}\right)\right| \leq B\left|p_{x}\left(x, \bar{s} ; \varepsilon_{r}\right)\right|,\left|f_{2}\left(x, \bar{s} ; \varepsilon_{r}\right)\right| \leq B\left[\left|p_{x x}\right|+\left|p_{x}\right|^{2}\right]\left(x, \bar{s} ; \varepsilon_{r}\right), x \in[0,1] .
$$


To estimate the function $\left|p_{x}\right|$, we use the integral equation (3.20). We have for $x \in(0,1)$

$$
p_{x}(x, s)=-\frac{\exp \left[-s\left(x-x_{0}\right)\right]}{2}+\frac{s^{2}}{2} \int_{0}^{1} \operatorname{sgn}(x-\xi) \exp (-s|x-\xi|)\left(\varepsilon_{r}(\xi)-1\right) p(\xi, s) d \xi .
$$

Hence, (2.1) and (3.3) imply that $\left|p_{x}\right| \leq B$ for $x \in[0,1]$. Since $\delta\left(x-x_{0}\right)=0$ for $x \in[0,1]$, then (3.3) implies that $p_{x x}=s^{2} \varepsilon_{r}(x) p$ for $x \in[0,1]$. Hence, by (2.1) and (3.3) $\left|p_{x x}\right| \leq B, x \in[0,1]$. Thus, (3.28) implies (3.25).

We now prove $(3.26)$. Let $\widetilde{p}(x, s)=p\left(x, \bar{s} ; \varepsilon_{r}^{(1)}\right)-p\left(x, \bar{s} ; \varepsilon_{r}^{(2)}\right)$. Then by (3.1)

$$
\widetilde{p}_{x x}-\bar{s}^{2} \varepsilon_{r}^{(1)}(x) \widetilde{p}=\bar{s}^{2}\left(\varepsilon_{r}^{(1)}-\varepsilon_{r}^{(2)}\right)(x) p\left(x, \bar{s} ; \varepsilon_{r}^{(2)}\right), x \in \mathbb{R} .
$$

In addition, it follows from (3.3) and (3.20) that functions $\partial_{x}^{j} \widetilde{p}(x, \bar{s}), j=0,1,2$ decay exponentially with $|x| \rightarrow \infty$. Hence, multiplying (3.29) by $\widetilde{p}$, integrating over $\mathbb{R}$ and using (2.1) and (3.3), we obtain $\|\widetilde{p}\|_{H^{1}(\mathbb{R})} \leq$ $B\left\|\varepsilon_{r}^{(1)}-\varepsilon_{r}^{(2)}\right\|_{L_{2}(0,1)}$. Hence, by (3.25) and (3.29) lead to

$$
\|\widetilde{p}\|_{H^{2}(0,1)} \leq B\left\|\varepsilon_{r}^{(1)}-\varepsilon_{r}^{(2)}\right\|_{L_{2}(0,1)} .
$$

This, (3.4) and (3.27) imply (3.26).

\section{Approximate Global Convergence.}

4.1. The concept. As to the 1-D version of that technique, which was published in [31] and which is used here, originally the work [31] was considered "only as a preliminary step before applying similar ideas to 2-D and 3-D cases" (page 125 of [31]). The authors of [31] meant the incorporation of the Quasi-Reversibility Method (QRM) in the technique of [5, 6, 7, 8, 9, 10, 11, 12, 27, 28, 32, 48], see section 5.

Least squares functionals for MCIPs with single measurement data suffer from multiple local minima and ravines. This implies the local convergence of conventional numerical methods for those MCIPs. The latter means that iterations should start from a point located in a sufficiently small neighborhood of the exact solution. The central question addressed in the above publications was: How to construct an effective numerical method, which would lead to a point in a sufficiently small neighborhood of the exact solution of an MCIP without any a priori information about that neighborhood? Furthermore, there should be a rigorous guarantee of reaching that neighborhood.

Because of the enormous challenge of addressing this central question (section 1), the approach of $[5,6,7,8,9,10,11,12,27,28,31,32,48]$ as well as of the current publication consists of the following six steps:

Step 1. A reasonable approximate mathematical model is proposed. The accuracy of this model cannot be rigorously estimated.

Step 2. A numerical method is developed, which works within the framework of this model.

Step 3. A theorem is proven, which guarantees that, within the framework of this model, the numerical method of Step 2 indeed reaches a sufficiently small neighborhood of the exact solution, as long as the error, both in the data and in some additional approximations is sufficiently small. It is central for our approach that this theorem should not rely neither on the assumption about a knowledge of any point in a small neighborhood of the exact solution nor on the assumption of a knowledge of the background medium inside of the domain of interest.

Step 4. That numerical method is tested on computationally simulated data.

Step 5 (optional). The numerical method of Step 2 is tested on experimental data. To have a truly unbiased case, blind data are preferable. This step is optional because it is usually not easy to actually get experimental data. 
Step 6. Finally, if results of Step 4 and (optionally) Step 5 are good ones, then that approximate mathematical model is proclaimed as a valid one.

Step 6 is logical, because its condition is that the resulting numerical method is proved to be effective, i.e. Goal 2 is successfully met, also see the fourth Remark 4.1. It is sufficient to achieve that small neighborhood of the exact solution after a finite (rather than infinite) number of iterations. Next, because of approximations in the mathematical model, the resulting solution can be refined via a locally convergent numerical method. The latter led to a two-stage numerical procedure in $[6,7,8,9,10,11,12,32,48]$. On the first stage the numerical method of Step 2 is applied. On the second stage the Adaptive Finite Element Method (adaptivity) for MCIPs takes the solution of the first stage as the starting point for iterations an refines it.

4.2. Definition. Considerations of subsection 4.1 led to the introduction of the approximate global convergence property in $[11,12]$. Since this definition is new, it is worthy to repeat it here. Recall that one of backbone principles of the theory of ill-posed problems is that, given an ill-posed problem, it is reasonable to assume uniqueness and existence of the exact solution of this problem for the "ideal" noiseless data $[11,22,26,47]$. It is not necessary that an algorithm addressing the above central question would start from any point. In fact, it is sufficient if it would start from such a reasonable point, which would not contain an information about a small neighborhood of the exact solution. The question of the validity of the approximate mathematical model $M$ of Definition 4.1 should be addressed in Steps 4,5 of subsection 4.1.

Definition 4.1 (approximate global convergence) [11, 12]. Consider a nonlinear ill-posed problem $P$. Suppose that this problem has a unique solution $x^{*} \in B$ for the noiseless data $y^{*}$, where $B$ is a Banach space with the norm $\|\cdot\|_{B}$. We call $x^{*}$ "exact solution" or "correct solution". Suppose that a certain approximate mathematical model $M$ is proposed to solve the problem $P$ numerically. Assume that, within the framework of the model $M$, this problem has unique exact solution $x_{M}^{*}$ and let $x_{M}^{*}=x^{*}$. Consider an iterative numerical method for solving the problem $P$. Suppose that this method produces a sequence of points $\left\{x_{n}\right\}_{n=1}^{N} \subset B$, where $N \in[1, \infty)$. Let a sufficiently small number $\theta \in(0,1)$. We call this numerical method approximately globally convergent of the level $\theta$, or shortly globally convergent, if, within the framework of the approximate model $M$, a theorem is proven, which guarantees that, without any a priori knowledge of a sufficiently small neighborhood of $x^{*}$, there exists a number $\bar{N} \in[1, N)$ such that

$$
\left\|x_{n}-x^{*}\right\|_{B} \leq \theta, \forall n \geq \bar{N} \text {. }
$$

Suppose that iterations are stopped at a certain number $k \geq \bar{N}$. Then the point $x_{k}$ is denoted as $x_{k}:=x_{g l o b}$ and is called "the approximate solution resulting from this method".

\section{Remarks 4.1:}

1. We repeat that we have introduced this definition because, in simple terms, nothing else works for MCIPs with single measurement data, see in section 1 comments about Goals 1,2.

2. The most important requirement of Definition 4.1 is that this numerical method should provide a sufficiently good approximation for the exact solution $x^{*}$ without any a priori knowledge of a sufficiently small neighborhood of $x^{*}$. Furthermore, one should have a rigorous guarantee of the latter, within the framework of the model $M$. In other words, Step 1 of subsection 4.1 should be addressed.

3. Unlike the classical convergence, this definition does not require $\lim _{n \rightarrow \infty} x_{n}=x^{*}$. Furthermore, the total number $N$ of iterations can be finite.

4. As to the use of the approximate mathematical model $M$, all equations of Mathematical Physics are approximate ones. The main criterion of their validity is the accuracy of descriptions of experimental data, which is exactly what we do. Also, it is well known that the Huygens-Fresnel optics is not yet rigorously derived from the Maxwell equations, see section 8.1 of the classical book of Born and Wolf [14]. Nevertheless it is the Huygens-Fresnel theory which describes the experimental data of the diffraction optics very well. Furthermore, the entire optical industry nowadays is based on the Huygens-Fresnel theory. On the other hand, the derivation of this theory from Maxwell equations is based on some non-rigorous approximations. Analogously, although the numerical method of works [5, 6, 7, 8, 9, 10,11, 12, 27, 28, 32, 48], is based on an approximate model, Goal 2 has been consistently achieved. 
5. Numerical Method. Although a detailed description of the numerical method of this section can be found in [31], we still present it rather briefly here in order to refer to some formulas in the convergence analysis in section 6. Also, because of $(2.2)$, we consider here the function $\ln \left(w / w_{0}\right)$ instead of $\ln w(x, s)$ in [31]. Let $w(x, s) \in C^{2+\alpha}\left(\left|x-x_{0}\right| \geq \beta\right) \cap C(\mathbb{R}), \forall \beta, s>0$ be the solution of the problem (2.8), (2.9), see Theorem 3.1.

5.1. Integral differential equation. Since by $(3.3) w(x, s)>0$, we can consider the function $r(x, s)$,

$$
r(x, s)=s^{-2}\left[\ln w(x, s)-\ln w_{0}(x, s)\right]=s^{-2} \ln \left[\left(w / w_{0}\right)(x, s)\right] .
$$

Then (2.8), (2.10) and (2.18) imply that

$$
\begin{gathered}
r_{x x}+s^{2} r_{x}^{2}-2 s r_{x}=\varepsilon_{r}(x)-1, x>0, \\
r(0, s)=\varphi_{0}(s), r_{x}(0, s)=\varphi_{1}(s), \\
\varphi_{0}(s)=s^{-2}[\ln \varphi(s)-\ln (2 s)]+x_{0} s^{-1}, \varphi_{1}(s)=2 / s-\exp \left(s x_{0}\right)\left(s^{2} \varphi(s)\right)^{-1} .
\end{gathered}
$$

By $(2.2),(2.5),(2.8)$ and $(2.9) w(x, s)=C(s) e^{-s x}$ for $x \geq 1$, where $C(s)$ is a certain function $s$-dependent on $s$. Hence,

$$
r_{x}(1, s)=0 .
$$

Differentiate equation (5.1) with respect to $s$. Then

$$
\begin{gathered}
q(x, s)=\partial_{s} r(x, s), \psi_{0}(s)=\varphi_{0}^{\prime}(s), \psi_{1}(s)=\varphi_{1}^{\prime}(s), \\
r(x, s)=-\int_{s}^{\bar{s}} q(x, \tau) d \tau+V(x, \bar{s}), \\
V(x, \bar{s})=\bar{s}^{-2}\left[\ln w(x, \bar{s})-\ln w_{0}(x, \bar{s})\right]=r(x, \bar{s}) .
\end{gathered}
$$

Here $\bar{s}>0$ is a sufficiently large number which is chosen in numerical experiments. We call $V(x, \bar{s})$ the tail function. Actually $\bar{s}$ is the regularization parameter of this method. Using (5.1)-(5.7), we obtain

$$
\begin{aligned}
q_{x x}-2 s^{2} q_{x} \int_{s}^{\bar{s}} q_{x}(x, \tau) d \tau & +2 s\left[\int_{s}^{\bar{s}} q_{x}(x, \tau) d \tau\right]^{2}-2 s q_{x}+2 \int_{s}^{\bar{s}} q_{x}(x, \tau) d \tau \\
& +2 s^{2} q_{x} V_{x}-4 s V_{x} \int_{s} q_{x}(x, \tau) d \tau+2 s\left(V_{x}\right)^{2}-2 V_{x}=0, s \in[\underline{s}, \bar{s}] \\
q(0, s) & =\psi_{0}(s), q_{x}(0, s)=\psi_{1}(s), q_{x}(1, s)=0, s \in[\underline{s}, \bar{s}] .
\end{aligned}
$$

Lemma 2.1 of [31] implies for $k=0,1,2$

$$
D_{x}^{k} r(x, s)=D_{x}^{k}\left\{-\frac{1}{s}\left[\int_{x_{0}}^{x} \sqrt{\varepsilon_{r}(\xi)} d \xi-\left(x-x_{0}\right)\right]+O\left(\frac{1}{s^{3}}\right)\right\}, x>0, s \rightarrow \infty .
$$


Hence, the function $V(x, \bar{s})=r(x, \bar{s})$ is small for large values of $\bar{s}$,

$$
\|V(x, \bar{s})\|_{C^{2}[0,1]}=O(1 / \bar{s}) .
$$

The main difficulty of this numerical method is in the solution of the problem (5.8), (5.9). Equation (5.8) has two unknown functions $q$ and $V$. To approximate both of them, we use a predictor/corrector-like scheme. First, given an approximation for $V$, we update $q$ via equation (5.8), (5.9). Next, we update the unknown coefficient $\varepsilon_{r}(x)$ and solve the forward problem $(2.8),(2.9)$ for $s:=\bar{s}$ with this updated coefficient $\varepsilon_{r}(x)$. This is our predictor-like step. On the corrector-like step we update the tail function $V(x, \bar{s})$ via (5.7).

Consider a partition of the interval $[\underline{s}, \bar{s}]$ into $N$ small subintervals with the grid step size $h>0$ and assume that the function $q(x, s)$ is piecewise constant with respect to $s$,

$$
\underline{s}=s_{N}<s_{N-1}<\ldots<s_{0}=\bar{s}, s_{i-1}-s_{i}=h ; q(x, s)=q_{n}(x) \text {, for } s \in\left(s_{n}, s_{n-1}\right] .
$$

Let $\mu>>1$ be a large parameter which should be chosen in numerical experiments. Multiply both sides of equation (5.8) by the Carleman Weight Function

$$
\mathcal{C}_{n, \mu}(s)=\exp \left[-\mu\left(s_{n-1}-s\right)\right], s \in\left(s_{n}, s_{n-1}\right]
$$

and integrate with respect to $s \in\left(s_{n}, s_{n-1}\right)$. The CWF is introduced in order to mitigate the influence of the nonlinear term $B_{n}(\mu, h)\left(q_{n}^{\prime}\right)^{2}$ in the resulting equation. If $h$ is fixed, then

$$
B_{n}(\mu, h)=O\left(\mu^{-1}\right), \mu \rightarrow \infty
$$

We ignore the nonlinear term, since we have observed in our computations that it provides only an insignificant impact in results for $\mu \geq 50$.. For each $n$ we perform inner iterations with respect to the tail function. This way we obtain functions $q_{n, k}, V_{n, k}$. The equation for the pair $\left(q_{n, k}, V_{n, k}\right)$ is

$$
\begin{gathered}
q_{n, k}^{\prime \prime}-\left(A_{1, n} h \sum_{j=0}^{n-1} q_{j}^{\prime}-A_{1, n} V_{n, k}^{\prime}-2 A_{2, n}\right) q_{n, k}^{\prime}=-A_{2, n} h^{2}\left(\sum_{j=0}^{n-1} q_{j}^{\prime}\right)^{2}+2 h \sum_{j=0}^{n-1} q_{j}^{\prime}+2 A_{2, n} V_{n, k}^{\prime}\left(h \sum_{j=0}^{n-1} q_{j}^{\prime}\right) \\
-A_{2, n}\left(V_{n, k}^{\prime}\right)^{2}+2 A_{2, n} V_{n, k}^{\prime}, q_{0}: \equiv 0, x \in(0,1) \\
q_{n, k}(0)=\psi_{0, n}, q_{n, k}^{\prime}(0)=\psi_{1, n}, q_{n, k}^{\prime}(1)=0 \\
\left.\psi_{0, n}=\frac{1}{h} \int_{s_{n}}^{s_{n-1}} \psi_{0}(s) d s, \psi_{1, n}=\frac{1}{h} \int_{s_{n}}^{s_{n-1}} \psi_{1}(s) d s .15\right)
\end{gathered}
$$

Here $A_{1, n}, A_{2, n}$ are certain numbers, whose exact expression is given in $[5,11]$. It is known that

$$
\max _{\mu h \geq 1}\left(A_{1, n}, A_{2, n}\right) \leq 8 \bar{s}^{2}
$$

5.2. The iterative process. Since equations (5.14) are generated by the Volterra-like integral differential equation (5.8), it is natural to solve them sequentially starting from $n=1$. Let $\xi \in(0,1)$ be a sufficiently small number. Consider a function $\chi(x) \in C^{2}(\mathbb{R})$ such that

$$
\chi(x)=\left\{\begin{array}{c}
1, x \in(\xi, 1-\xi), \\
\text { between } 0 \text { and } 1 \text { for } x \in(0, \xi) \cup(1-\xi, 1), \\
0, x \in \mathbb{R} \backslash(0,1) .
\end{array}\right.
$$


The existence of such functions is well known from the Real Analysis course. We choose the first guess for the tail function $V_{0}(x)$ as in subsection 6.3. For each $n \in[1, N]$ we perform $m$ iterations with respect to tails. Hence, we obtain three finite sequences of functions:

$$
\left\{q_{n, k}(x)\right\}_{(n, k)=(1,1)}^{(N, m)},\left\{V_{n, k}(x)\right\}_{(n, k)=(1,1)}^{(N, m)},\left\{\varepsilon_{r}^{(n, k)}(x)\right\}_{(n, k)=(1,1)}^{(N, m)}, x \in[0,1] .
$$

Step $n^{(1)}, n \in[1, N]$. Suppose that functions $q_{j}(x) \in H^{4}(0,1), V_{n-1}(x) \in C^{3}[0,1]$ are constructed. We set $V_{n, 1}(x):=V_{n-1}(x)$. Next, using the QRM (subsection 5.3), we approximately solve equation (5.14) for $k=1$ with over-determined boundary conditions (5.15) and find the function $q_{n, 1} \in H^{4}(0,1)$ this way (Remark 5.1). Hence, by the embedding theorem $q_{n, 1} \in C^{3}[0,1]$. Next, we find the approximation $\varepsilon_{r}^{(n, 1)}$ for the unknown coefficient $\varepsilon_{r}(x)$ via the following two formulas

$$
\begin{gathered}
r_{n, 1}(x)=-h q_{n, 1}-h \sum_{j=0}^{n-1} q_{j}+V_{n, 1}, x \in[0,1], \\
\bar{\varepsilon}_{r}^{(n, 1)}(x)=1+r_{n, 1}^{\prime \prime}(x)+s_{n}^{2}\left[r_{n, 1}^{\prime}(x)\right]^{2}-2 s_{n} r_{n, 1}^{\prime}(x), x \in[0,1], \\
\varepsilon_{r}^{(n, 1)}(x)=\left\{\begin{array}{c}
\bar{\varepsilon}_{r}^{(n, 1)}(x), \text { if } \bar{\varepsilon}_{r}^{(n, 1)}(x) \in[1, d], x \in[0,1], \\
1, \text { if } \bar{\varepsilon}_{r}^{(n, 1)}(x)<1, x \in[0,1], \\
d, \text { if } \bar{\varepsilon}_{r}^{(n, 1)}(x)>d, x \in[0,1] .
\end{array}\right.
\end{gathered}
$$

Hence, $\bar{\varepsilon}_{r}^{(n, 1)} \in C^{1}[0,1]$ and $\varepsilon_{r}^{(n, 1)} \in C^{\alpha}[0,1], \forall \alpha \in(0,1)$. Formulas (5.20) and (5.21) are obvious discrete analogs of formulas (5.1) and (5.6) respectively. Consider the function

$$
\widehat{\varepsilon}_{r}^{(n, 1)}(x)=(1-\chi(x))+\chi(x) \varepsilon_{r}^{(n, 1)}(x), x \in \mathbb{R} .
$$

It can be easily proven that $\widehat{\varepsilon}_{r}^{(n, 1)}(x) \in[1, d]$ for $x \in \mathbb{R}$. This implies the ellipticity of the operator (2.8) for

$$
\varepsilon_{r}(x):=\widehat{\varepsilon}_{r}^{(n, 1)}(x) \in C^{\alpha}(\mathbb{R}) .
$$

Hence, we solve the forward problem $(2.8),(2.9),(5.24)$ for $s:=\bar{s}$. Let $w_{n, 1}(x, \bar{s})$ be the solution of this problem considered in Theorem 3.1. Then, using (5.7), we set for the next tail

$$
V_{n, 2}(x):=\frac{\ln w_{n, 1}(x, \bar{s})-\ln w_{0}(x, \bar{s})}{\bar{s}^{2}} .
$$

Step $n^{(k)}, n \in[1, N], k \in[2, m]$. Suppose that functions $q_{j}(x) \in H^{4}(0,1), j \in[0, n-1]$,

$V_{n, k}(x) \in C^{2+\alpha}[0,1]$ are constructed. Using the QRM, we approximately solve the problem equation (5.14), (5.15). This gives us the function $q_{n, k} \in H^{4}(0,1) \subset C^{3}[0,1]$. Next, we find the approximation $\varepsilon_{r}^{(n, k)} \in C^{\alpha}[0,1]$ for the function $\varepsilon_{r}(x)$, using formulas (5.20)-(5.22), where the index "1" is replaced with the index " $k$ ". Suppose now that $n<N$. Then we construct the function $\widehat{\varepsilon}_{r}^{(n, k)}(x)$ via $(5.23)$, where $(n, 1)$ is replaced with $(n, k)$ and solve the forward problem $(2.8),(2.9)$ with $\varepsilon_{r}(x):=\widehat{\varepsilon}_{r}^{(n, k)}(x)$ at $s:=\bar{s}$. If $k<m$, then define the tail $V_{n, k+1}(x)$ by an obvious analog of (5.25). If, however, $n<N$ and $k=m$, then we set

$$
V_{n+1}(x):=V_{n, m+1}(x) \in C^{3}[0,1], q_{n}(x):=q_{n, m}(x)
$$

and go to the step $(n+1)^{(1)}$. If $n=N$ and $k=m$, then we set $\varepsilon_{r, g l o b}(x):=\varepsilon_{r}^{(N, m)} \in C^{\alpha}[0,1]$ and stop the process. The function $\varepsilon_{r, g l o b}(x)$ is our final solution as defined in Definition 4.1. 
5.3. The Quasi-Reversibility Method (QRM). The QRM was first proposed in [34]. We refer to, e.g. $[15,18,30]$ and references cited there for some further developments. The QRM can solve linear ill-posed boundary value problems for many PDEs, including problems with over-determined boundary conditions. It finds least squares solutions. Let

$$
a_{n, k}(x)=A_{1, n} h \sum_{j=0}^{n-1} q_{j}^{\prime}-A_{1, n} V_{n, k}^{\prime}-2 A_{2, n},
$$

and $H_{n, k}(x)$ be the right hand side of equation (5.14). Then $a_{n, k} \in C^{2}[0,1], H_{n, k} \in L_{2}(0,1)$. Equation (5.14) can be rewritten as

$$
L_{n, k}\left(q_{n, k}\right):=q_{n, k}^{\prime \prime}-a_{n, k} q_{n, k}^{\prime}=H_{n, k} .
$$

Let $\gamma \in(0,1)$ be the regularization parameter. In the QRM we minimize the following Tikhonov functional with respect to the function $q_{n, k}$ subject to boundary conditions (5.15),

$$
J_{\gamma}\left(q_{n, k}, H_{n, k}\right)=\left\|L_{n, k}\left(q_{n, k}\right)-H_{n, k}\right\|_{L_{2}(0,1)}^{2}+\gamma\left\|q_{n, k}\right\|_{H^{4}(0,1)}^{2},
$$

Remark 5.1. There is a peculiarity of this technique in the 1-D case. We use approximate least squares QRM solutions of the problem (5.14), (5.15). On the other hand, it seems to be at the first glance that for a given function $V_{n, k}$ one can find the function $q_{n, k}$ via solving equation (5.14) with only two out of three boundary conditions in (5.15). However, our attempt to do so for computationally simulated data has failed to produce good quality results, see Remark 3.1 on page 130 of [31]. This is because of the approximate nature of our mathematical model, see subsection 6.3.

Lemma 5.1 (Carleman estimate, see [31] and p. 168 in [30]). For any function $u \in H^{2}(0,1)$ with $u(0)=u^{\prime}(0)=0$ and for any parameter $\lambda \geq 1$ the following Carleman estimate holds

$$
\int_{0}^{1}\left(u^{\prime \prime}\right)^{2} e^{-2 \lambda x} d x \geq \frac{1}{16} \int_{0}^{1}\left[8\left(u^{\prime \prime}\right)^{2}+\lambda\left(u^{\prime}\right)^{2}+\lambda^{3}(u)^{2}\right] e^{-2 \lambda x} d x .
$$

Lemma 5.2 [31]. For any number $\gamma \in(0,1)$ and for each function $F \in L_{2}(0,1)$ there exists unique minimizer $u_{\gamma} \in H^{4}(0,1)$ with $u_{\gamma}(0)=u_{\gamma}^{\prime}(0)=0$ of the functional $J_{\gamma}(u, F)$ in (5.27) and

$$
\left\|u_{\gamma}\right\|_{H^{4}(0,1)} \leq C \gamma^{-1 / 2}\|F\|_{L_{2}(0,1)}
$$

where the constant $C>0$ is independent on $a_{n, k}, F, u_{\gamma}, \gamma$. Let $\left\|a_{n, k}\right\|_{C[0,1]} \leq a_{0}$, where $a_{0}=$ const. $>0$. Let the function $u \in H^{4}(0,1), L_{n, k}(u)=H$ and $u(0)=u^{\prime}(0)=0$. Then there exists a constant $K=K\left(a_{0}\right)>0$ independent on $F, H, u_{\gamma}, u, \gamma$ such that

$$
\left\|u_{\gamma}-u\right\|_{H^{2}(0,1)} \leq K\left(\|F-H\|_{L_{2}(0,1)}+\sqrt{\gamma}\|u\|_{H^{4}(0,1)}\right) .
$$

The proof of Lemma 5.2 is based on Lemma 5.1, Riesz theorem and the variational principle. The main difference between Lemma 5.1 and Carleman estimates for elliptic operators in the $n-\mathrm{D}(n \geq 2)$ case is that we now have the integration in the right hand side of $(5.28)$ over the entire interval $(0,1)$ rather than over a subdomain of the original domain in $n-\mathrm{D}$ [35]. The absence of local minima of the functional (5.27) follows from Lemma 5.2. It can be derived from Lemma 5.1 that it is not necessary to use the regularization term $\gamma\left\|q_{n, k}\right\|_{H^{4}(0,1)}^{2}$ in (5.27). Still, we use this term to ensure that functions $\varepsilon_{r}^{(n, k)} \in C^{1}[0,1]$. Although Lemma 5.1 implies that it is not necessary to use the condition $q_{n, k}^{\prime}(1)=0$, we use it in our computations to improve the stability.

\section{Convergence Analysis.}


6.1. The exact solution. Using again the concept of Tikhonov for ill-posed problems [11, 22, 26, 47], we assume that there exists unique exact solution $\varepsilon_{r}^{*}(x)$ of our CIP with the "ideal" noiseless data $g^{*}(t)$ in (2.6), and the function $\varepsilon_{r}^{*}(x)$ satisfies conditions $(2.1),(2.2)$. Then $\varepsilon_{r}^{*}(x)$ generates functions, which are defined similarly with the ones above, $q_{0}^{*} \equiv 0$,

$$
r^{*}(x, s), V^{*}(x, \bar{s}):=r^{*}(x, \bar{s}), q^{*}(x, s)=\partial_{s} r^{*}(x, s), q_{n}^{*}(x), \psi_{0, n}^{*}, \psi_{1, n}^{*}, n \in[1, N] .
$$

Similarly with (5.14), (5.15) we obtain from (5.8) and (5.9)

$$
\begin{gathered}
\partial_{x}^{2} q_{n}^{*}-\left(A_{1, n} h \sum_{j=0}^{n-1} \partial_{x} q_{j}^{*}-A_{1, n} \partial_{x} V^{*}-2 A_{2, n}\right) \partial_{x} q_{n}^{*}=-A_{2, n} h^{2}\left(\sum_{j=0}^{n-1} \partial_{x} q_{j}^{*}\right)^{2}+2 h \sum_{j=0}^{n-1} \partial_{x} q_{j}^{*} \\
+2 A_{2, n} \partial_{x} V^{*}\left(h \sum_{j=0}^{n-1} \partial_{x} q_{j}^{*}\right)-A_{2, n}\left(\partial_{x} V^{*}\right)^{2}+2 A_{2, n} \partial_{x} V^{*}+F_{1, n}(x, \mu, h, \bar{s}), x \\
\in(0,1), \\
q_{n}^{*}(0)=\psi_{0, n}^{*}, \partial_{x} q_{n}^{*}(0)=\psi_{1, n}^{*}, \partial_{x} q_{n, k}^{*}(1)=0 .
\end{gathered}
$$

In (6.2) $F_{n}(x, \mu, h)$ is the error function which is generated by averaging (5.16) of boundary functions $\psi_{0}^{*}, \psi_{1}^{*}$. Also, the nonlinear term $B_{n}(\mu, h)\left(\partial_{x} q_{n}^{*}\right)^{2}$ is included in $F_{1, n}$. By embedding theorem there exists a constant $C_{2} \geq 1$ such that

$$
\|f\|_{C[0,1]} \leq C_{2}\|f\|_{H^{1}(0,1)}, \forall f \in H^{1}(0,1), .
$$

Let a given number $C^{*}>0$ be such that

$$
\max _{n \in[1, N]}\left\|q_{n}^{*}\right\|_{C^{1}[0,1]} \leq \frac{C^{*}}{C_{2}}, \quad \max _{n \in[1, N]}\left\|q_{n}^{*}\right\|_{H^{4}(0,1)} \leq C^{*} .
$$

6.2. Approximate mathematical model. Following Definition 4.1, we introduce in this subsection an approximate mathematical model. Assumptions of this model are based on the asymptotic formulas (5.10) and (5.11). These assumptions actually mean that we consider only the first term of the asymptotic behavior of the tail function $V^{*}(x, \bar{s})$ when the pseudo frequency $\bar{s} \rightarrow \infty$ and truncate the rest. We call $\bar{s}$ the truncation pseudo frequency. A similar assumption is used in, e.g. geometrical optics. Our Approximate Mathematical Model consists of the following two

\section{Assumptions:}

1. Let the parameter $\bar{s}>1$. Then there exists a function $a^{*} \in H^{4}(0,1)$ such that the function $V^{*}(x, s)$ has the form

$$
V^{*}(x, s)=s^{-1} a^{*}(x), \forall s \geq \bar{s}, \forall x \in[0,1] .
$$

2. The following equality holds

$$
\bar{s}^{-1} a^{*}(x)=\bar{s}^{-2}\left[\ln w^{*}(x, \bar{s})-\ln w_{0}(x, \bar{s})\right] .
$$

Compare (6.6), (6.7) with (5.7), (5.11). Using the third condition (6.1) and (6.7), we obtain

$$
q^{*}(x, \bar{s})=-\bar{s}^{-2} a^{*}(x) .
$$

Consider equation (5.8) for the pair $\left(q^{*}(x, s), V^{*}(x, \bar{s})\right), s \in[\underline{s}, \bar{s}]$. Then boundary conditions (5.9) are valid with functions $\psi_{0}^{*}(s), \psi_{1}^{*}(s)$. Hence, substituting in (5.8), (5.9) $s:=\bar{s}$ and using (6.8), we obtain that the function $a^{*}(x)$ is the solution of the following over-determined boundary value problem

$$
\partial_{x}^{2} a^{*}(x)=0, x \in(0,1)
$$




$$
a^{*}(0)=-\bar{s}^{2} \psi_{0}^{*}(\bar{s}), \partial_{x} a^{*}(0)=-\bar{s}^{2} \psi_{1}^{*}(\bar{s}), \partial_{x} a^{*}(1)=0 .
$$

In (6.10) exact boundary data at $x=0$ are used. In practice, however we have the non-exact data boundary $\psi_{0}(s), \psi_{1}^{*}(s)$. Hence, consider the following boundary value problem for the function $a(x)$

$$
\begin{gathered}
\partial_{x}^{2} a(x)=0, x \in(0,1), \\
a(0)=-\bar{s}^{2} \psi_{0}(\bar{s}), \partial_{x} a(0)=-\bar{s}^{2} \psi_{1}(\bar{s}), \partial_{x} a(1)=0 .
\end{gathered}
$$

We solve the problem (6.11), (6.12) via the QRM (see Remark 5.1). Let the function $a_{\gamma} \in H^{4}(0,1)$ be the unique minimizer of the following analog of the QRM functional (5.27)

$$
\bar{J}_{\gamma}(a)=\left\|a^{\prime \prime}\right\|_{L_{2}(0,1)}^{2}+\gamma\|a\|_{H^{4}(0,1)}^{2},
$$

subject to boundary conditions (6.12). Because of (6.6)-(6.13), we define the first guess for the tail function as

$$
V_{0}(x)=\bar{s}^{-1} a_{\gamma}(x) .
$$

Let $\sigma \in(0,1)$ be the level of the error in the boundary data. We assume that

$$
\left|\psi_{0}(\bar{s})-\psi_{0}^{*}(\bar{s})\right|+\left|\psi_{1}(\bar{s})-\psi_{1}^{*}(\bar{s})\right| \leq \sigma .
$$

Remarks 6.1.

1. Let $a_{\gamma}(x)$ be the approximate solution of the problem (6.11)- (6.13). Then substituting (6.14) in (5.1), (5.6) at $s=\bar{s}$, one can find a good approximation for the exact coefficient $\varepsilon_{r}^{*}(x)$. Furthermore, Theorem 6.1 implies that all functions $\varepsilon_{r}^{(n, k)}$ are good approximations for $\varepsilon_{r}^{*}(x)$, as long as the total number of iterations is not too large. This corresponds well with (4.1). Since we find $a_{\gamma}(x)$ only using the boundary data, then this means that our approximate mathematical model is indeed a good one. Hence, we can stop iterations on any function $\varepsilon_{r}^{(n, k)}$ for those indices $(n, k)$, which are "allowed" by Theorem 6.1. Next, one can use the adaptivity procedure to refine the solution (the end of subsection 4.1). This was confirmed numerically in tests 2,3 of [6] as well as in tests 2,3 in section 4.16.2 of [11]. However, if not using the adaptivity for refinement, then, quite naturally, one needs to find an optimal iteration number to stop, see Figures 7.3, 7.5, 7.6 and 7.8 of [5], Figures 3,4 in [9], Figures 6 in [27] and Figure 1-b) in [31] (this again corresponds well with Definition 4.1). These figures can also be found in chapters 3-5 of the book [11], along with objective stopping criteria for iterations.

2. Because of the approximate nature of equalities (6.6), (6.7), equation (6.9) does not match the asymptotic behavior (5.10), which is the single self-contradiction of this approximate mathematical model. The same can be stated about all other versions of this method in above cited publications. Nevertheless, it was consistently demonstrated that this numerical method works well for both computationally simulated and experimental data. Based on our numerical experience, we believe that this is because of two factors: (1) The truncation of the asymptotic series with respect to $1 / \bar{s}$ at $\bar{s} \rightarrow \infty$ in (6.6) is reasonable, and (2) The use of the predictor-corrector procedure to update tails via solutions of forward problems.

Lemma 6.1. For each value of the regularization parameter $\gamma \in(0,1)$ there exists unique minimizer $a_{\gamma} \in H^{4}(0,1)$ of the functional (6.13) satisfying boundary conditions (6.12). The following estimates hold

$$
\left\|a_{\gamma}\right\|_{H^{4}(0,1)} \leq C \bar{s}^{2} \gamma^{-1 / 2}\left(\left|\psi_{0}(\bar{s})\right|+\left|\psi_{1}(\bar{s})\right|\right),
$$

where the constant $C>0$ is independent on $a_{\gamma}, \gamma, \bar{s}, \psi_{0}(\bar{s}), \psi_{1}(\bar{s})$. Let Assumptions 1,2 hold and the function $a^{*} \in H^{4}(0,1)$ be the solution of the problem (6.9), (6.10). Let (6.15) holds. Then there exists a constant $K>0$ independent on $a^{*}(x), a_{\gamma}(x), \gamma, \bar{s}, \psi_{0}(\bar{s}), \psi_{1}(\bar{s})$ such that

$$
\left\|V_{0}-V^{*}\right\|_{H^{2}(0,1)} \leq K \bar{s}\left(\sigma+\sqrt{\gamma}\left\|a^{*}\right\|_{H^{4}(0,1)}\right) .
$$


This lemma easily follows from Lemma 5.2, (5.5) and (6.6)-(6.14). Uniqueness within the framework of this Approximate Mathematical Model can be easily derived, see Lemma 6.6.2 in [11] for a similar result. We assume below that the above exact solution $\varepsilon_{r}^{*}(x)$ is exactly the same as the one within the framework of this model, i.e.

$$
\varepsilon_{r}^{*}(x)=r_{x x}^{*}+s^{2}\left(r_{x}^{*}\right)^{2}-2 s r_{x}^{*}+1, s \in[\underline{s}, \bar{s}], x \in[0,1],
$$

see (5.1). Here the function $r^{*}$ is defined via (5.6), (5.7), where functions $q, V$ are replaced with functions $q^{*}(x, s), V^{*}(x, \bar{s})$, and (6.6)-(6.8) hold. Hence, (6.2)-(6.4) and (6.16) imply the following analogs of discrete formulas (5.20), (5.21)

$$
\begin{gathered}
r_{n}^{*}(x)=-h q_{n}^{*}(x)-h \sum_{j=0}^{n-1} q_{j}^{*}(x)+V^{*}(x, \bar{s})+F_{2, n}(x, h, \bar{s}), x \in[0,1], \\
\varepsilon_{r}^{*}(x)=1+\partial_{x}^{2} r_{n}^{*}(x)+s_{n}^{2}\left[\partial_{x} r_{n}^{*}(x)\right]^{2}-2 s_{n} \partial_{x} r_{n}^{*}(x)+F_{3, n}(x, h, \bar{s}), x \in[0,1] .
\end{gathered}
$$

One can prove that

$$
\sum_{j=1}^{3}\left\|F_{j, n}\right\|_{L_{2}(0,1)} \leq C_{1} \bar{s}^{2}\left(h+\mu^{-1}\right),
$$

where the constant $C_{1}>0$ is independent on $h, \mu, \bar{s}$. We assume that

$$
F_{j, n} \equiv 0, j=1,2,3 ; \psi_{0, n}=\psi_{0, n}^{*}, \psi_{1, n}=\psi_{1, n}^{*}, n \in[1, N] .
$$

Therefore the by (6.15) error is "allowed" only in numbers $\psi_{0}^{*}(\bar{s}), \psi_{1}^{*}(\bar{s})$. We point out that an analog of Theorem 6.1 can be proved very similarly for the realistic case (6.19) and when $\left|\psi_{0, n}-\psi_{0, n}^{*}\right|,\left|\psi_{1, n}-\psi_{1, n}^{*}\right|<$ $\sigma$. So, condition (6.20) is introduced for brevity only. An additional simplification of the presentation comes from balancing error parameters $h, \sigma$ with the regularization parameter $\gamma$ and parameters $\xi, \mu$ in $(5.12)$ and (5.18) as

$$
\sigma=\sqrt{\gamma}=\sqrt{2 \xi}=\mu^{-1}:=h
$$

6.3. Approximate global convergence theorem. The main new element of Theorem 6.1 is that we iteratively estimate tails, which was not done in [31]. To do this, we use (3.25) and (3.26). The proof of Theorem 6.1 has some similarities with the proof of Theorem 6.7 of [11] for the 3-D case. However, estimates of the $H^{2}$-norm of the QRM solution $q_{n, k}$ in [11] are obtained in a subdomain of the domain $\Omega$. In turn, this leads to the estimate of the accuracy of the computed target coefficient in another subdomain of $\Omega$ rather than in the entire $\Omega$. On the othe hand, because of the 1-D case, (5.28) implies a stronger estimate of that accuracy in the whole interval $(0,1)$. Still, because of the above similarity with the proof of Theorem 6.7 of [11], and also because the proof of Theorem 6.1 is rather technical, we only outline it here.

Theorem 6.1. (approximate global convergence). Let the function $\varepsilon_{r}^{*}(x)$ satisfying conditions (2.1), (2.2) be the exact solution of our CIP for the noiseless data $g^{*}(t)$ in (2.6). Fix the truncation pseudo frequency $\bar{s}>\max \left(\widehat{s}\left(d,\left|x_{0}\right|\right), 1\right)$, where the number $\widehat{s}\left(d,\left|x_{0}\right|\right)$ was defined in Theorem 3.1. Let two Assumptions of subsection 6.2 hold and $\left\|a^{*}\right\|_{H^{4}(0,1)} \leq C^{*}$, where $C^{*}$ is the constant defined in (6.5). Let the first tail function $V_{0}(x)$ be defined via (6.11)-(6.14). In addition, let conditions (6.15), (6.20) and (6.21) hold. Let $B=B\left(x_{0}, d, \bar{s}\right)>1, C_{2}, C^{*}$ and $K$ be the numbers defined in Theorem 3.2, (6.4), (6.5) and Lemma 6.1. Define $B_{1}=B_{1}\left(x_{0}, d, \bar{s}\right)=\max \left(C_{2}, B, C^{*}, K \bar{s}\right)$. Consider the iterative process of subsection 5.2. Let the number $N \geq 2$ be independent on $h$. Then there exists a constant $D=D\left(x_{0}, d, \bar{s}\right) \geq 32 \bar{s}^{2} B_{1}$ such that if for a constant $b>1$ independent on $x_{0}, d, \bar{s}$

$$
h \in\left(0, h_{0}\right), h_{0}=\frac{1}{b D^{2 N m+2}},
$$


then the following estimates are valid

$$
\begin{gathered}
\left\|q_{n, k}-q_{n}^{*}\right\|_{H^{2}(0,1)} \leq D^{(n-1) m+k+2} h, \\
\left\|q_{n, k}\right\|_{C^{1}[0,1]} \leq 2 C^{*}, \\
\left\|\varepsilon_{r}^{(n, k)}-\varepsilon_{r}^{*}\right\|_{L_{2}(0,1)} \leq D^{(n-1) m+k+1} h, \\
\left\|V_{n, k}^{\prime}-\partial_{x} V^{*}\right\|_{L_{2}(0,1)}+\left\|V_{n, k}^{\prime \prime}-\partial_{x}^{2} V^{*}\right\|_{L_{2}(0,1)} \leq D^{(n-1) m+k+1} h, \\
\left\|V_{n, k}^{\prime}\right\|_{C[0,1]} \leq 2 B_{1}, \\
\left|A_{1, n} h \sum_{j=0}^{n-1} q_{j}^{\prime}-A_{1, n} V_{n, k}^{\prime}-2 A_{2, n}\right|_{C[0,1]} \leq 18 \bar{s}^{2} B_{1}:=a_{0} .
\end{gathered}
$$

Here $q_{n, k}$ are QRM solutions of boundary value problems (5.14), (5.15). In particular, let the number $\omega \in(0,1)$ be defined as

$$
\omega=\frac{\ln b}{\ln b+(2 N m+2) \ln D} .
$$

Then (6.25) implies the Hölder-like estimate

$$
\left\|\varepsilon_{r}^{(n, k)}-\varepsilon_{r}^{*}\right\|_{L_{2}(0,1)} \leq h^{\omega}:=\theta
$$

which guarantees the approximate global convergence property of the level $\theta$ of this iterative process within the framework of the approximate mathematical model of subsection 6.2 (Definition 4.1).

\section{Remarks 6.2.}

1. Since the number $N$ of $s$-subintervals $\left(s_{i}, s_{i-1}\right)$ is assumed to be independent on the partition step size $h$, then Theorem 6.1 requires the length of the total $s$-interval $[\underline{s}, \bar{s}]$ covered in the iterative process of subsection 5.2 to decrease with the decrease of $h$. This seems to be a natural requirement. Indeed, if the number $\bar{s}-\underline{s}$ would be independent on $h$, then this would mean the increase of the number of iteration $N m=m \cdot(\bar{s}-\underline{s}) / h$. On the other hand, the error increases with the iteration number, especially for nonlinear ill-posed problems. Hence, the number of iterations $N m$ is one of regularization parameters here. It was pointed out on pages 156, 157 of the book [22] that the total number of iterations can often be regarded as a regularization parameter in the theory of Ill-Posed Problems. Two other regularization parameters are $\bar{s}$ and $\gamma$. Thus, we have a vector $(N m, \bar{s}, \gamma)$ of regularization parameters.

2. Another argument justifying the smallness of the interval $[\underline{s}, \bar{s}]$ is that the original equation (5.8) contains Volterra-like integrals in nonlinear terms. It is well known from the standard ODE course that existence of solutions of Volterra-like integral equations is guaranteed only on sufficiently small intervals.

Outline of the proof of Theorem 6.1. To avoid using new notations, below $q_{n, k}(x)$ means the QRM solution of the problem (5.14), (5.15). The only exception is when we subtract (6.2) and (6.3) from (5.14), (5.15) respectively. Denote

$$
\widetilde{q}_{n, k}=q_{n, k}-q_{n}^{*}, \widetilde{\varepsilon}_{r}^{(n, k)}=\varepsilon_{r}^{(n, k)}-\varepsilon_{r}^{*}, \widetilde{V}_{n, k}=V_{n, k}-V^{*} .
$$


Consider the case $(n, k)=(1,1)$. First, by Lemma 6.1

$$
\left\|\widetilde{V}_{1,1}^{\prime}\right\|_{L_{2}(0,1)}+\left\|\widetilde{V}_{1,1}^{\prime \prime}\right\|_{L_{2}(0,1)} \leq B_{1} h<D^{2} h .
$$

Hence, (3.25), (6.4), (6.22) and (6.29) imply that

$$
\left\|V_{1,1}^{\prime}\right\|_{C[0,1]}=\left\|\widetilde{V}_{1,1}^{\prime}+\partial_{x} V^{*}\right\|_{C[0,1]} \leq C_{2} D^{2} h+B_{1} \leq 2 B_{1} .
$$

Estimates (6.29) and (6.30) establish (6.26) and (6.27) for $(n, k)=(1,1)$. Consider now the function $\widetilde{q}_{1,1}$. Subtracting (6.2) from (5.14) and (6.3) from (5.15), using (6.20) and setting $n=k=1$, we obtain

$$
\begin{gathered}
\widetilde{q}_{1,1}^{\prime \prime}+\left(A_{1,1} V_{1,1}^{\prime}+2 A_{2,1}\right) \widetilde{q}_{1,1}^{\prime}=-\left[A_{1,1} \partial_{x} q_{1}^{*}+A_{2,1}\left(V_{1,1}^{\prime}+\partial_{x} V^{*}\right)\right] \widetilde{V}_{1,1}, x \in(0,1), \\
\widetilde{q}_{1,1}(0)=\widetilde{q}_{1,1}(0)=\widetilde{q}_{1,1}^{\prime}(1)=0 .
\end{gathered}
$$

We now need to estimate the QRM solution $\bar{q}_{1,1}$ of the problem (6.31), (6.32). By (5.17), (6.29) and (6.30)

$$
\left|A_{1,1} V_{1,1}^{\prime}+2 A_{2,1}\right| \leq 8 \bar{s}^{2}\left(B_{1}+2\right) \leq \frac{1}{2} a .
$$

Next, using (3.25), (5.17), (6.5), (6.29) and (6.30), we obtain

$$
\begin{gathered}
\left|A_{1,1} \partial_{x} q_{1}^{*}+A_{2,1}\left(V_{1,1}^{\prime}+\partial_{x} V^{*}\right)\right| \leq 8 \bar{s}^{2}\left(C^{*}+3 B_{1}\right) \leq 32 \bar{s}^{2} B_{1}, \\
\left\|\left[A_{1,1} \partial_{x} q_{1}^{*}+A_{2,1}\left(V_{1,1}^{\prime}+\partial_{x} V^{*}\right)\right] \widetilde{V}_{1,1}^{\prime}\right\| \leq 32 \bar{s}^{2} B_{1}^{2} h \leq D^{2} h .
\end{gathered}
$$

We can take $D \geq K\left(a_{0}\right)$, where numbers $a_{0}$ and $K\left(a_{0}\right)$ are defined in (6.33) and Lemma 5.2 respectively. Hence, Lemma 5.2, (6.31), (6.32) and (6.34) imply that

$$
\left\|\widetilde{q}_{1,1}\right\|_{H^{2}(0,1)} \leq D^{3} h,
$$

which proves (6.23) for $\widetilde{q}_{1,1}$. Next, to prove (6.24), we use (6.5), (6.22) and (6.35),

$$
\left\|q_{1,1}\right\|_{C^{1}[0,1]} \leq C_{2}\left\|q_{1,1}\right\|_{H^{2}(0,1)} \leq C_{2}\left\|\widetilde{q}_{1,1}\right\|_{H^{2}(0,1)}+C_{2}\left\|q_{1}^{*}\right\|_{H^{2}(0,1)} \leq 2 C^{*} .
$$

Next, subtract (6.18) from (5.21). Note that by (2.1) and (5.22) $\left|\widetilde{\varepsilon}_{r}^{(n, k)}(x)\right|=\left|\varepsilon_{r}^{(n, k)}(x)-\varepsilon_{r}^{*}(x)\right| \leq$ $\left|\bar{\varepsilon}_{r}^{(n, k)}(x)-\varepsilon_{r}^{*}(x)\right|, \forall x \in[0,1]$. Hence, using (6.22), (6.29), (6.30), (6.35) and (6.36), we obtain

$$
\begin{gathered}
\left\|\widetilde{\varepsilon}_{r}^{(1,1)}(x)\right\|_{L_{2}(0,1)} \leq\left(D^{3} h^{2}+B_{1} h\right)\left(1+\bar{s}^{2}\left(3 C^{*} h+3 B_{1}+2\right)\right) \\
\leq 7 \bar{s}^{2}\left(D^{3} h^{2}+B_{1} h\right) \leq 14 \bar{s}^{2} D h \leq D^{2} h,
\end{gathered}
$$

which establishes (6.25) for $n=k=1$. Next, using (3.26), (5.18), (5.23) and (6.21), we obtain

$$
\left\|\widetilde{V}_{1,2}^{\prime}\right\|_{L_{2}(0,1)}+\left\|\widetilde{V}_{1,2}^{\prime \prime}\right\|_{L_{2}(0,1)} \leq 2 B D^{2} h+\sqrt{2 \xi} d \leq D^{3} h .
$$

Hence, similarly with (6.30) $\left\|V_{1,2}^{\prime}\right\| \leq 2 B_{1}$. Thus, (6.26) and (6.27) are established for $n=1, k=2$. The rest of the proof can be done similarly using the mathematical induction. We need (6.28) to estimate norms $\left\|\widetilde{q}_{n, k}\right\|_{H^{2}(0,1)}$ via Lemma 5.2. The estimate (6.28) is proved using (5.17), (6.22) and (6.24). 


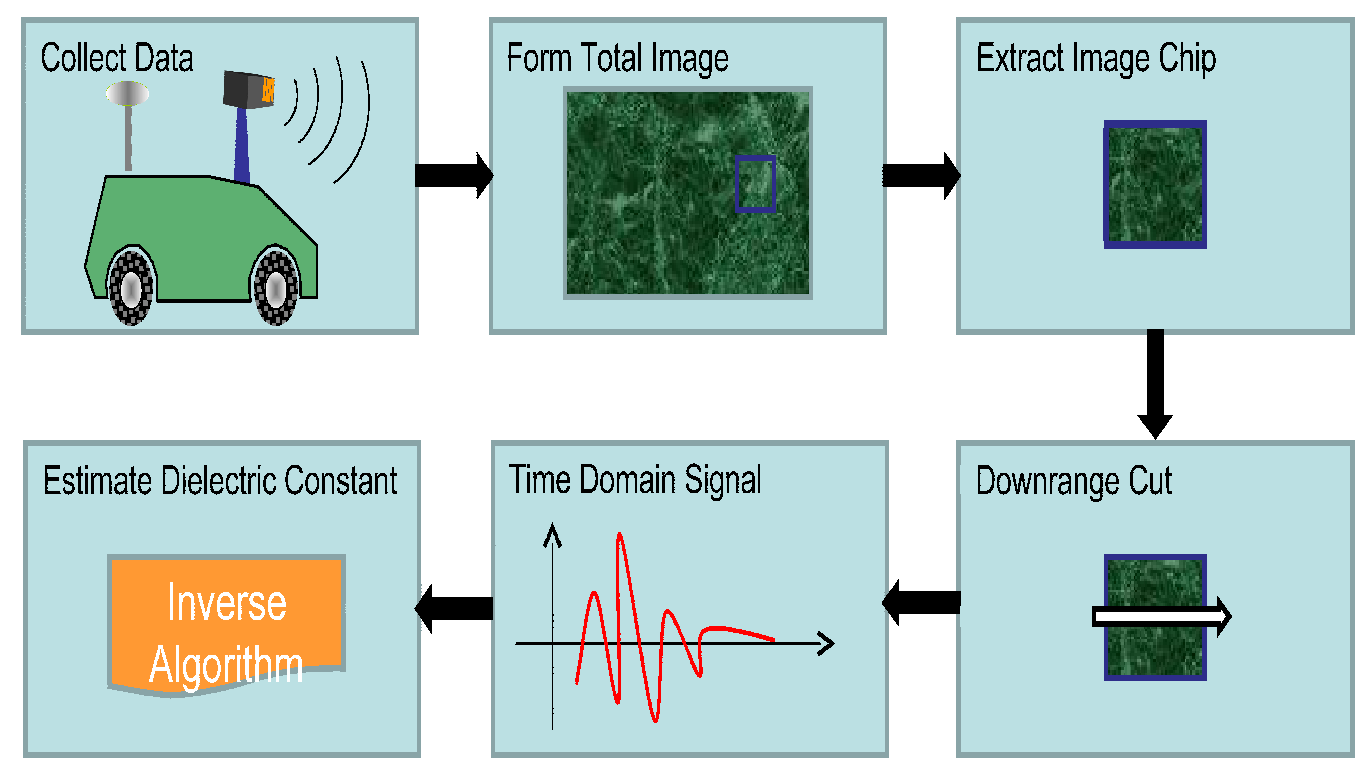

Fig. 7.1: The schematic diagram of data collection by the Forward Looking Radar of US Army Research Laboratory.

\section{Experimental Setup and Data Pre-Processing.}

7.1. Data collection. The schematic diagram of the data collection by the Forward Looking Radar is depicted on Figure 7.1. The time resolved electric pulses are emitted by two sources installed on the radar. Only one component of the electric field is both originated and measured in the backscattering regime. The data are collected by sixteen (16) detectors with the step size in time of 0.133 nanosecond. Only shallow targets placed both below and above the ground can be detected. The depth of the upper part of a shallow underground target is a few centimeters. The Ground Positioning System (GPS) provides the distance between the radar and a point on the ground located above that target. The error in the latter is a few centimeters. Time resolved voltages of backreflected signals are integrated over radar/target distances between 20 meters and 8 meters, and they are also averaged with respect to both source positions as well as with respect to readings of sixteen detectors. Since the radar/target distance is known, then it is approximately known which part of the measured time resolved signal corresponds to the reflections from that target, see Figure 7.1. However, clutter obviously obscures a significant part to that signal. For any target of interest, only a single time dependent curve can be extracted from the vast amount of data, see samples on Figures 7.4-b), d) ,f). This is the curve we have worked with in each of available five cases of experimental data.

Since the radar/target distance is provided by GPS with a good accuracy, geometrical parameters of 
targets, including their depths, are not of an interest here. The main goal of our work was to provide to calculate ratios $R$ of dielectric constants

$$
R=\frac{\varepsilon_{r}(\text { target })}{\varepsilon_{r}(\text { bckgr })}
$$

where $\varepsilon_{r}(\mathrm{bckgr})$ is the dielectric constant of the background medium. If $\varepsilon_{r}$ (bckgr) is known, then (7.1) enables to calculate $\varepsilon_{r}$ (target). If a target is located above the ground, then $\varepsilon_{r}$ (bckgr $)=\varepsilon_{r}($ air $)=1$. Since targets can be mixtures of constituent materials, then $\varepsilon_{r}$ (target) is a certain weighted average of dielectric constants of these materials. We image the ratio (7.1) rather than the function $\varepsilon_{r}(x)$ itself, since (2.1) requires that $\varepsilon_{r}(\mathrm{bckgr})$ should have a constant value outside of our domain of interest $x \in(0,1)$. The latter was true only for targets located above the ground. However, in the case of underground targets, this condition is invalid because of the air/ground interface. Below "1" stands for "1 meter".

A separate question is about the meaning of the dielectric constant in metallic targets. Comparison of Figures 7.2-a) and 7.2-b) shows that the electric field reflected from the metallic target is about the same as the one reflected from the dielectric target with a large value of the dielectric constant. Next, we have simulated the data for four large inclusion/background contrasts via solving the problem (2.8), (2.9). Figure 7.2-c) shows computed functions $w(0, s)-w_{0}(0, s), s \in[1,12]$ for the case of a single inclusion embedded in the interval $(0,1)$ with four different values of the function $\varepsilon_{r}=10,20,30,40$ in this inclusion, see Figure 7.3a) for the geometry. One can observe that these curves do not change much with the change of $\varepsilon_{r} \in[10,30]$. Furthermore, curves for $\varepsilon_{r}=30,40$ are almost the same. Therefore, based on Figures 7.2-a)-c), we choose the interpretation (7.2) of the dielectric constant in metals. The physical meaning of (7.2) is unknown, and we call it appearing dielectric constant for metals,

$$
\varepsilon_{r}(\text { metal }) \in[10,30] .
$$

7.2. Main uncertainties in the experimental data. To figure out what kind of ideal data one should expect for the case of one target only, we have performed computational simulations via solving the forward problem $(2.3),(2.4)$ for the case of one target and for the source position

$$
x_{0}=-1 .
$$

In data simulation we have replaced $\mathbb{R}$ in $(2.3)$ with the interval $x \in(-6,6)$ and have considered zero Dirichlet boundary conditions for rather small times,

$$
u(-6, t)=u(6, t)=0, t \in(0,4) .
$$

Condition (7.4) is imposed because the wavefront originated at the source position (7.3) does not reach points $x= \pm 6$ for $t \in(0,4)$. The structure of the medium and the computed function $u(0, t):=g(t)$ are depicted on Figures 7.3-a) and 7.3-b), respectively. In the case $\varepsilon_{r}$ (target) $=$ const. $\in(0,1)$ the function $g(t)$ looks similar (not shown), except that its peak points downwards. Note that the constant background on Figures $7.3-\mathrm{a}), \mathrm{b}$ ) corresponds to the fundamental solution of the 1-D wave equation $v_{t t}=v_{x x}$,

$$
u_{0}(x, t)=\frac{1}{2} H\left(t-\left|x-x_{0}\right|\right),
$$

where $H$ is the Heaviside function. Figures 7.4-b), d), f) show the experimental data for different targets. A visual comparison of Figures 7.4-b), d), f) with Figure 7.3-b), confirms the above mentioned (section 1) substantial discrepancy between computationally simulated and experimental data. This discrepancy is the main challenge of working with these data.

In addition to the above misfit and the 3-D reality versus only a single curve for each target, there were some other uncertainties here as well. The most significant uncertainties were:

1. The reference signal was not measured. 


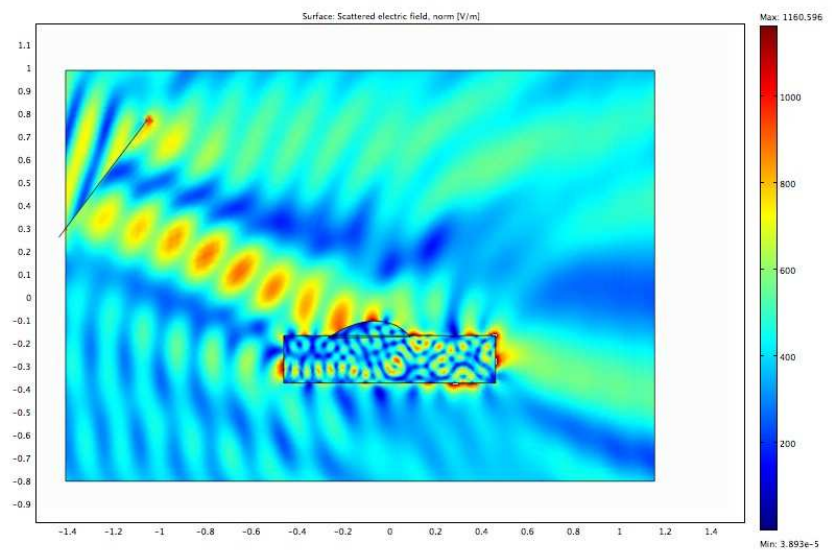

a)

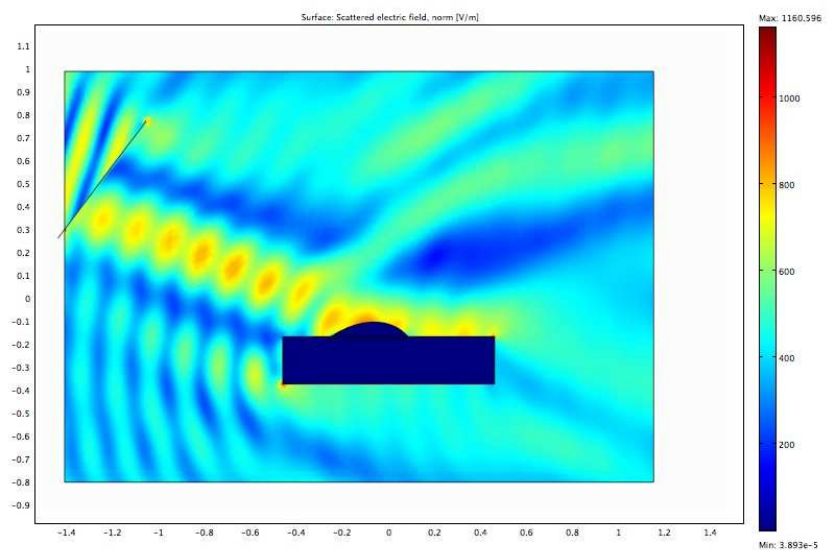

b)

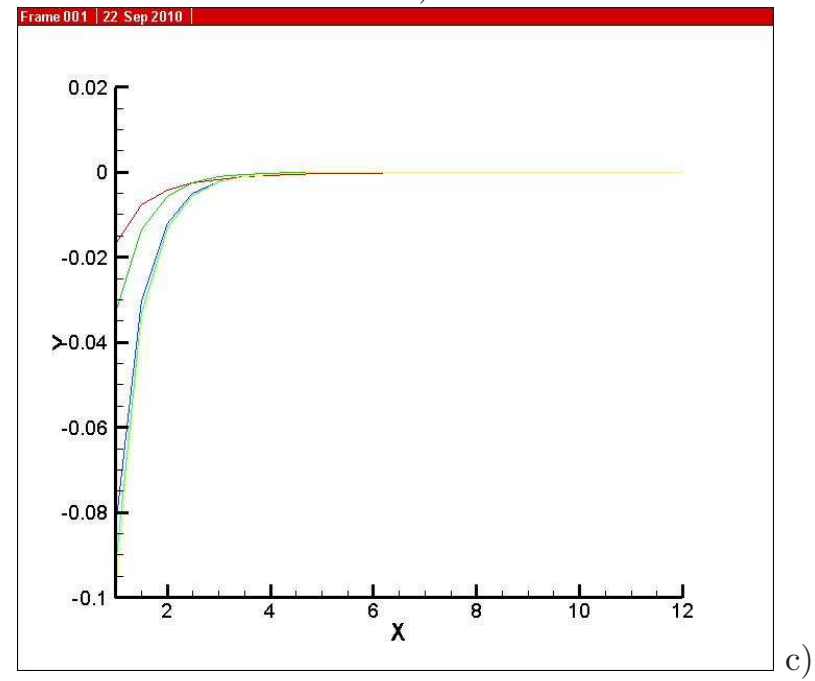

Fig. 7.2: Appearing dielectric constant for metals for computationally simulated data at a single frequency. a) The amplitude of the electric field reflected from a dielectric target with $\varepsilon_{r}=10$ in it. b) The amplitude of the electric field reflected from a metallic target on the same frequency as one on a). Reflected fields a) and b) are very similar. c) Computed functions $\left(w-w_{0}\right)(0, s), s \in[1,12]$ for the case when one inclusion is embedded in the interval $(0,1)$ with different values of the function $\varepsilon_{r}$, see Figure 7.3-a) for the location of the inclusion. From top to bottom $\varepsilon_{r}=10,20,30,40$. Curves for $\varepsilon_{r}=30,40$ almost coincide. Hence, the function $w(0, s)$ does not change much with the increase of the inclusion/background contrast from 10 to 40. Figures a)-c) justify the definition (7.2) of the appearing dielectric constant for metals. 


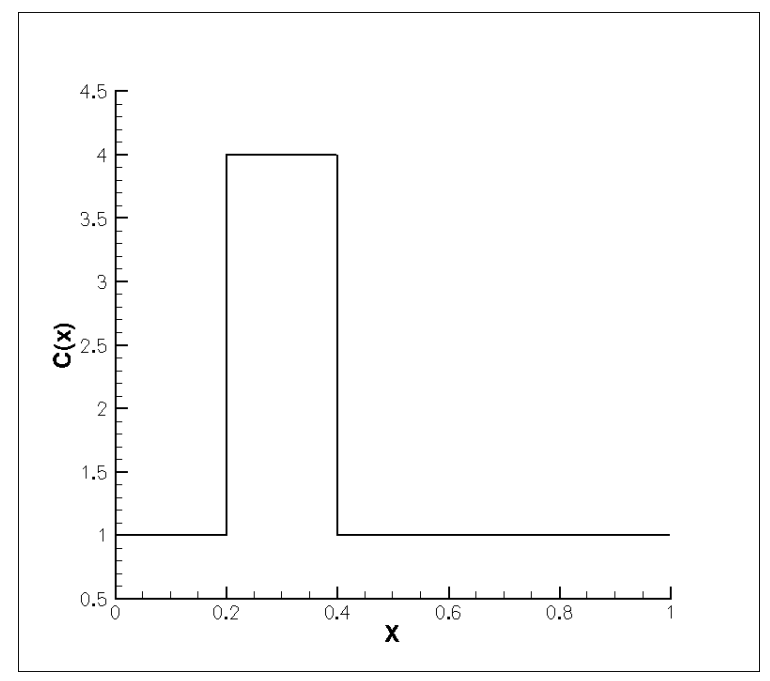

a)

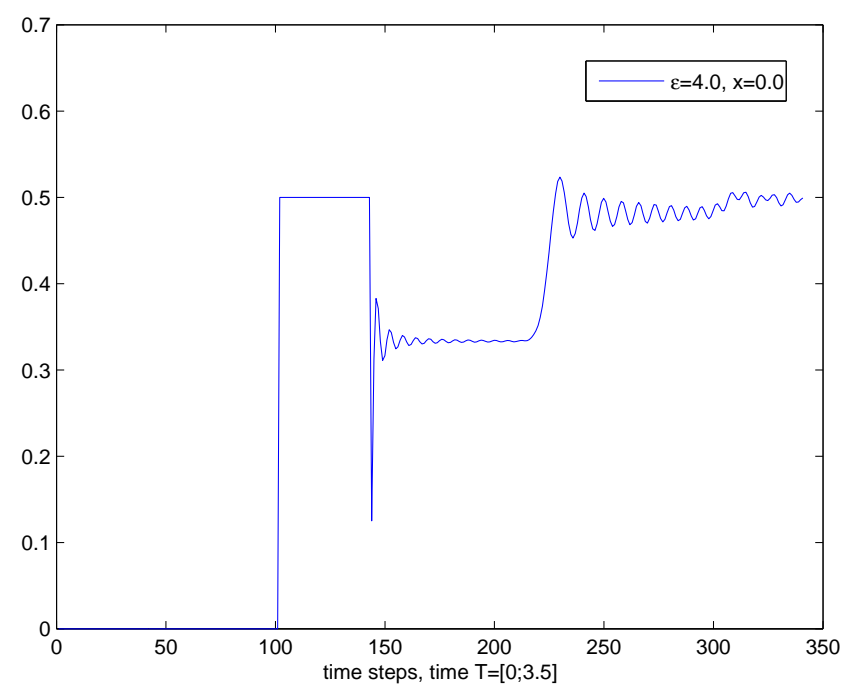

b)

Fig. 7.3: Computationally simulated data $u(0, t)$, where $u(x, t)$ is the solution of the problem (2.1), (2.2) with the source location $x_{0}=-1$. Boundary conditions (7.4) were used, since the wavefront did not yet reach points $x= \pm 6$ for times $t \in(0,4)$. a) The function $\varepsilon_{r}(x), x \in(0,1)$. Also, $\varepsilon_{r}(x)=1$ for $x \notin(0,1)$. b) The function $u(0, t)$ in time $T=[0,3.5]$.

2. The direction of the incident plane wave was oblique to the ground rather than orthogonal, see Figures 7.4-a), c), e).

3. Units for the amplitude of experimental data were unknown.

4. The location of the point source $x_{0}$ was unknown. Thus, (7.3) is an intuitive choice.

5. The time moment $t=0$ on the data was unknown.

6. The background was heterogeneous due to clutter.

Remark 7.1. It is because of items 4 and 5 that it was impossible to image locations of targets correctly.

At the same time, the UNCC/ChalmersGU team had the following two pieces of the information in advance:

1. The presence of a single target for each data set.

2. It was known whether the target was located above or below the ground.

7.3. Data pre-processing. We need to pre-process the experimental data in such a way that the resulting time resolved curves would look similarly with Figure 7.3-b), since this would fit, at least somehow, our mathematical model. If the target is located above the ground, then $\varepsilon_{r}$ (target) $>1$, since $\varepsilon_{r}(\mathrm{bckgr})=\varepsilon_{r}($ air $)=$ 1 in this case. Figure 7.3-b) indicates that one should select on the experimental curve only one downwards looking peak in this case. However, if the target is buried in the ground, then there could be any relation between $\varepsilon_{r}$ (target) and $\varepsilon_{r}$ (bckgr). Hence, based on Figures 7.3-a), b) as well as their analogs for the case $\varepsilon_{r}$ (target) $\varepsilon_{r}$ (bckgr) (not shown), we have selected on each experimental curve the earliest peak of the largest amplitude out of all other peaks. The rest of each curve was set to zero. More precisely, our selection of that peak was as follows: this should be the earliest peak of the largest amplitude

$$
\text { out of }\left\{\begin{array}{c}
\text { all peaks for a target buried in the ground, } \\
\text { all downwards looking peaks for a target above the ground. }
\end{array}\right.
$$


Bush (Clutter, Test 2)

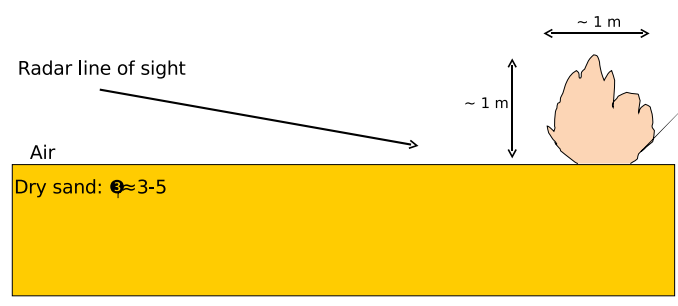

Target is desert bush seen along the road.

a)

Wood Stake (Test 3)

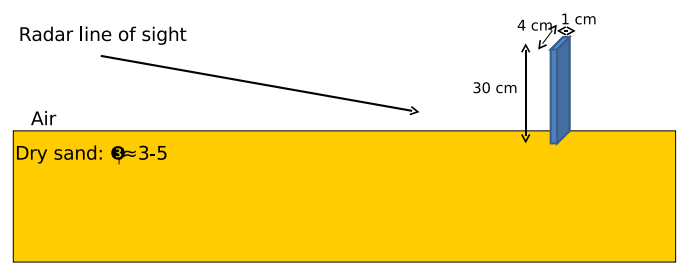

Target is wood stake on the ground.

c)

Flush Buried Metal Box (Test 4)

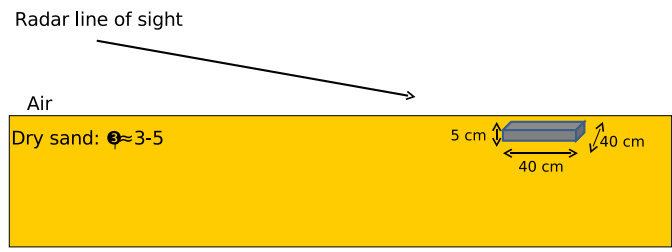

Target is flush buried metal box.

e)

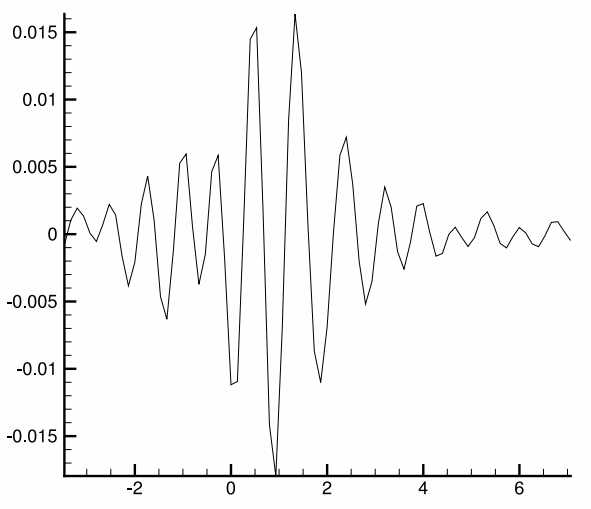

b)

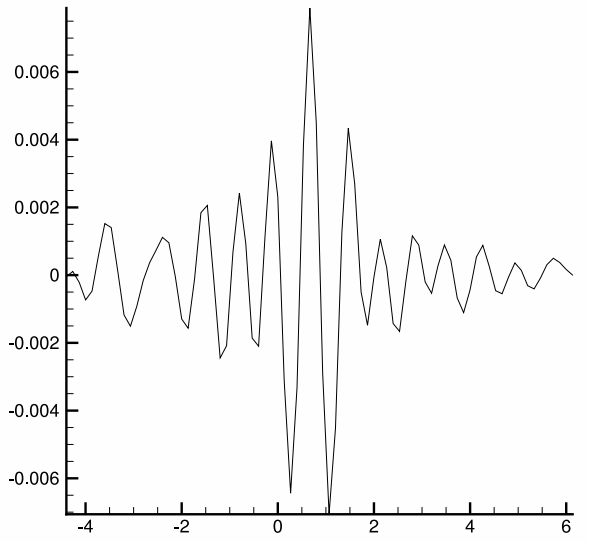

d)

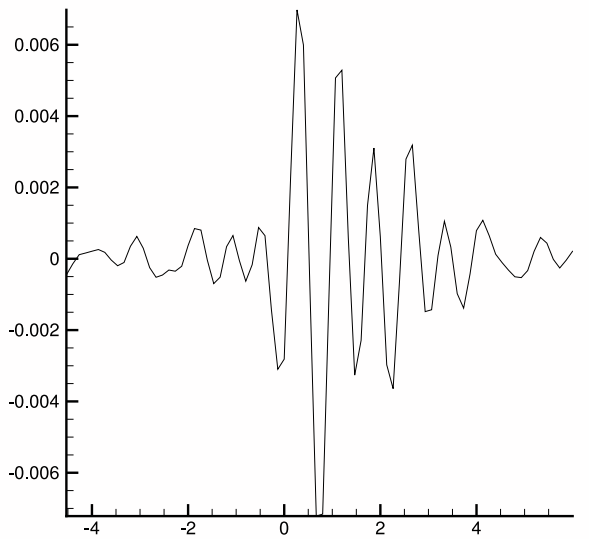

f)

Fig. 7.4: Targets and experimental data for Tests 1-3. The ground is dry sand with $\varepsilon_{r} \in[3,5]$ [45]. Because of the blind study case, a), c), e) were released to the UNCC/ChalmersGU team only after computations were made. a) Bush standing along the road (clutter). b) Scaled experimental data for a) (subsection 7.3). The horizontal axis is time in nanoseconds with the time step 0.133 nanosecond. The vertical axis is the amplitude of the measured voltage. c) Wood stake. d) Scaled experimental data for c). e) 23 Metal box buried in the dry sand. f) Scaled experimental data for e). The amplitude of largest downward looking peak on $f$ ) is 0.0072, whereas the amplitude of the largest upwards looking peak is 0.00\%. Therefore, that downwards looking peak was used in our data pre-processing. A huge misfit between experimental and computationally simulated data is evident: compare highly oscillatory curves of b), d), f) with Figure 7.3-b). Waveforms of b), d), f) show why the radar detection and discrimination problem is so challenging. One can see three very different types of targets, yet their signatures are very similar. 


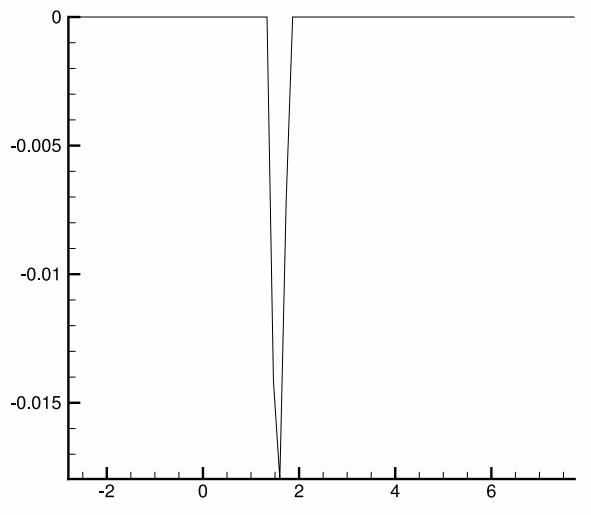

a)

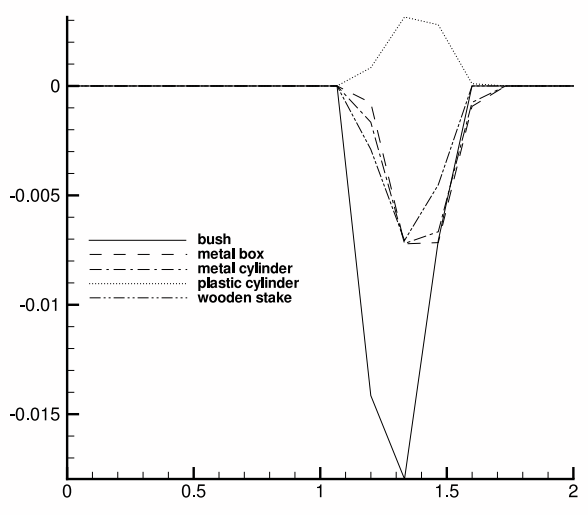

b)

Fig. 7.5: The horizontal axis is time in nanoseconds with the time step size of 0.133 nanosecond. a) The preprocessed experimental data for the bush standing along the road (clutter), see Figures 7.4-a),b). b) The pre-processed experimental data for all five cases (superimposed). In terms of our mathematical model, we use these curves as functions $u(0, t)-u_{0}(0, t)$.

We have assigned on each experimental curve the time zero $\{t=0\}$ to be such a point on the time axis, which is one (1) nanosecond off to the left from the beginning of the selected peak. Next, we have multiplied the resulting data by the scaling factor (below) and have regarded the resulting curve as the pre-processed data. Figure 7.5-a) displays the pre-processed data for the case of Figure 7.4-b). Figure 7.5-b) shows superimposed pre-processed curves for all five cases of experimental data we have possessed.

The measured amplitude for each case was of the order of $10^{5}$. This is well above the amplitude of Figure 7.3-b). Hence, we have multiplied all signals by the same scaling factor $10^{-7}$. We have chosen this number since the amplitudes of the Laplace transform of the resulting pre-processed data are about the same as values of the function $w(0, s)-w_{0}(0, s)$ for simulated solutions $w(x, s)$ of the problem $(2.8)$, (2.9) with inclusion/background contrasts in $\varepsilon_{r}$ ranging from 2 to 40, see Figure 7.2-c).

7.4. Functions $\psi_{0}(s)$ and $\psi_{1}(s)$. Let $\widetilde{g}(t)$ be the pre-processed experimental data for any of our targets. Based on Figures 7.5, we have calculated the Laplace transform $(2.7) \varphi(s)=\mathcal{L}(g)$ via the integration over the interval $t \in(0,2)$. Next, we have calculated the derivative $\varphi^{\prime}(s)$ as

$$
\varphi^{\prime}(s)=-\int_{0}^{2} \widetilde{g}(t) t e^{-s t} d t+\partial_{s} w_{0}(0, s),
$$

where the function $w_{0}(0, s)$ is defined in (2.11). It was observed in computational simulations of [31] that the function $\varphi(s)$ has the best sensitivity to the presence of inclusions for $s \in[0.5,1.2]$. Nevertheless, we have observed that the larger interval $s \in[1,12]$ provides better quality images for simulated data. The function $\varphi(s)$ was computed accurately for the entire interval $s \in[1,12]$ with the step size $\Delta s=0.05$ in the $s$-direction. However, because of their dependence on the derivative $\varphi^{\prime}(s)$ in (7.5), functions $\psi_{0}(s)=$ $q(0, s), \psi_{1}(s)=q_{x}(0, s)$ have oscillated for $s \in[3,12]$. On the other hand, our testing of computationally simulated data has shown that oscillations should not be present.

Hence, we have pre-processed the function $\psi_{0}(s)$ as follows. First, we have calculated $\psi_{0}(s)$ for $s \in[1,2.5]$ using the Laplace transform of the data, like in (7.5), with the 0.5 step size with respect to $s$. Next, we have set $\psi_{0}(12):=0.025 \cdot \psi_{0}(2.5)$. Next, we have linearly interpolated in the plane $\left(s, \psi_{0}\right)$ between points 
$\left(2.5, \psi_{0}(2.5)\right)$ and $\left(12, \psi_{0}(12)\right)$. And similarly for $\psi_{1}(s)$. Having functions $\psi_{0}(s), \psi_{1}(s)$ for our pre-processed experimental data, we have calculated numbers $\psi_{0, n}, \psi_{1, n}$ in (5.16), which are used as boundary conditions for the QRM.

8. Imaging Results. A detailed description of the numerical implementation of the algorithm of subsections 5.2, 5.3 can be found in [31]. We now briefly outline main elements as well as some differences with [31]. Unlike [31], were only synthetic data were used, the values $q_{n}(1)$ were unknown to us now (naturally). But this turned out not to be a problem. Indeed, our numerical experiments with computationally simulated data have shown to us that the knowledge of the numbers $q_{n}(1)$ affects only the accuracy of the image of the location of a target. However, it does not affect the accuracy of the reconstruction of the target/background contrast in $\varepsilon_{r}$, which was our main goal, see Remark 7.1. Thus, unlike [31], we have not assigned any values to $q_{n}(1)$. The initial tail function was taken as in (6.14). Although Theorem 6.1 guarantees approximate global convergence only for the case of a small $s$ - interval, our computational experience tells us that the interval $s \in[1,12]$ is an optimal one. We attribute this to the well known fact that convergence estimates are rotinely much more pessimistic than computational results. This is because constants in convergence theorems usually are largely over-estimated. Similarly, although the above theory works only for the case $\varepsilon_{r}(x) \geq 1$, this did not prevent us from computing one case whith $R<1$ in (7.1).

We regard $R:=R(x)$ in (7.1) as $x$-dependent function. With respect to the results of this section, $\varepsilon_{r}(x)$ in (2.8) was replaced with $R(x)$. Thus, it is $R(x)$ which was computed by the above algorithm. Let $R_{\text {comp }}(x)$ be the computed coefficient $R(x)$. Then we define the computed target/background contrast as $\bar{R}=\max R_{\text {comp }}(x)$ in the case when $\max R_{\text {comp }}(x)>1$, and as $\bar{R}=\min R_{\text {comp }}(x)$ in the case when $\max R_{\text {comp }}(x) \leq 1$. We set $\varepsilon_{r}$ (target) $:=\bar{R} \cdot \varepsilon_{r}$ (bckgr) .

For each test we have also computed two curves: the Laplace transform of the pre-processed experimental data and the function $w(0, s)$, where $w(0, s)$ is the solution of the problem (2.8), (2.9) with $\varepsilon_{r}(x):=$ $R_{\text {comp }}(x)$. The interval $s \in[1,12]$ with the step size $h=0.5$ was used for the latter. These these two curves were very close to each other for all tests.

Test 1. Computationally simulated data. First, we verify that our algorithm provides an accurate target/background contrast for computationally simulated data. We image the structure depicted on Figure 7.3-a). Figure 8.1-a) displays the resulting image. Recall that we are interested in accurate imaging of target/background contrasts rather than in accurate imaging of locations of targets (subsection 7.1). The imaged target/background contrast is 3.8 , whereas the real contrast is 4 . Thus, the imaged contrast is quite accurate. This gives us a hope that contrasts for experimental data are also computed accurately.

Test 2. Image of a bush, see Figures 7.4-a), 7.5-a), b). This was the most difficult case, because the target was highly heterogeneous one. Besides, the maximal of the modulus of the amplitude of the preprocessed signal for this target exceeds this values for other targets by the factor of 2.57. Figures 8.1-b) and 8.1-c) display the resulting image and the above superimposed curves respectively. Only a small difference between curves of Figure 7.4-c) is observed.

Test 3. Image of a wood stake, see Figures 7.4-c), 7.5-b). The computed image is displayed on Figure 8.1-d).

Test 4. Image of a metal box, see Figures 7.4-e), 7.5-b). The computed image is displayed on Figure 8.1-e).

Since we had five pieces of data in our possession, we have also imaged two more cases: plastic cylinder and metal box, both buried in soil. Dielectric constants were not measured when the data were collected. Therefore, we have compared computed values of dielectric constants with those listed in Tables [45, 46]. Note that these tables often provide a range of values rather than exact numbers. Soil was dry sand, where the dielectric constant varies between 3 and 5 [45]. Denote $\varepsilon_{r, p u b l}$ (target) the published value of the dielectric constant for non-metallic targets. By $(7.2)$ we regard $\varepsilon_{r, p u b l}$ (target) $\in[10,30]$ for metals. The case of bush (vegetation) is not listed in [45, 46]. Hence, we took $\varepsilon_{r, p u b l}$ (target) for vegetation from Figures 2,3 of [20]. Table 8.1 summarizes our results.

\section{Conclusions.}




\begin{tabular}{|c|c|c|c|c|c|}
\hline Target & $a / b$ & $\bar{R}$ & $\varepsilon_{r}(\mathrm{bckgr})$ & $\varepsilon_{r, \mathrm{comp}}($ target $)$ & $\varepsilon_{r, p u b l}($ target $)$ \\
\hline Fig. 6-a) & $\mathrm{n} / \mathrm{a}$ & 3.8 & 1 & 3.8 & 4, Fig. 6-a) \\
\hline bush & $\mathrm{a}$ & 6.4 & 1 & 6.4 & $\in[3,20]$, see $[20]$ \\
\hline wood stake & $\mathrm{a}$ & 3.8 & 1 & 3.8 & $\in[2,6]$, see $[45]$ \\
\hline metal box & $\mathrm{b}$ & 3.8 & $\in[3,5]$, see $[45]$ & $\in[11.4,19]$ & $\in[10,30]$, see $(7.2)$ \\
\hline metal cylinder & $\mathrm{b}$ & 4.3 & $\in[3,5]$, see $[45]$ & $\in[12.9,21.4]$ & $\in[10,30]$, see $(7.2)$ \\
\hline plastic cylinder & $\mathrm{b}$ & 0.28 & $\in[3,5]$, see $[45]$ & $\in[0.84,1.4]$ & 1.2, see $[46]$ \\
\hline
\end{tabular}

Table 8.1: Blindly computed values $\bar{R}$ for five targets, $\varepsilon_{r, \text { comp }}$ (target) $:=\bar{R} \cdot \varepsilon_{r}($ bckgr $)$. Here "a/b" means "above/below the ground". For "a" and "b" the background is air and dry sand, respectively.

Since dielectric constants were not measured in experiments, then the maximum what can be done is to compare computational results with tabulated values. Therefore, the most important conclusion from Table 8.1 is that blindly computed dielectric constants of targets fall well within tabulated limits. This was achieved regardless on a severely limited information content, significant discrepancies between experimental and computationally simulated data and some other significant uncertainties (subsection 7.2). Naturally, these factors have resulted in a large and yet unknown noisy component in the data which we have used as an input for our algorithm. Thus, we conclude that these results point towards both the adequacy of our mathematical model and a high degree of robustness of our numerical method.

Detailed studies of larger experimental data sets are necessary to figure out accuracy boundaries of our technique for this specific application. The authors hope to do so in the future.

\section{Acknowledgments}

This research was supported by US Army Research Laboratory and US Army Research Office grant W911NF-11-1-0399, the Swedish Research Council, the Swedish Foundation for Strategic Research (SSF) in Gothenburg Mathematical Modelling Centre (GMMC) and by the Swedish Institute, Visby Program. The authors are grateful to Drs. Paul Sacks and Natee Pantong for a number of useful discussions. 


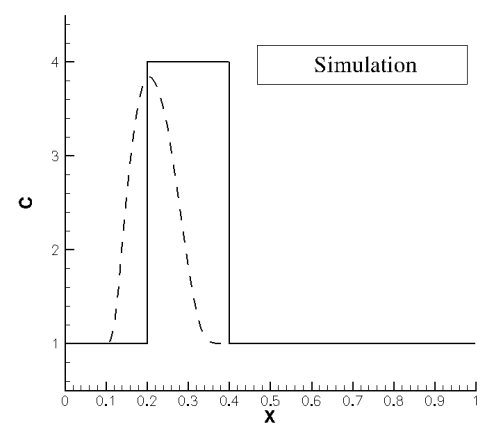

a)

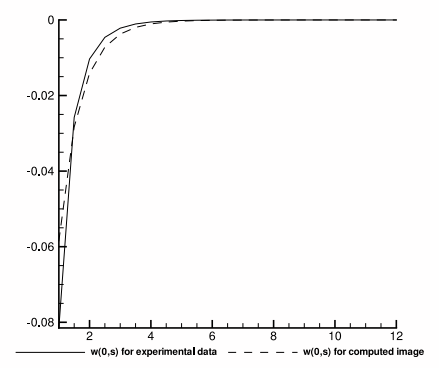

c)

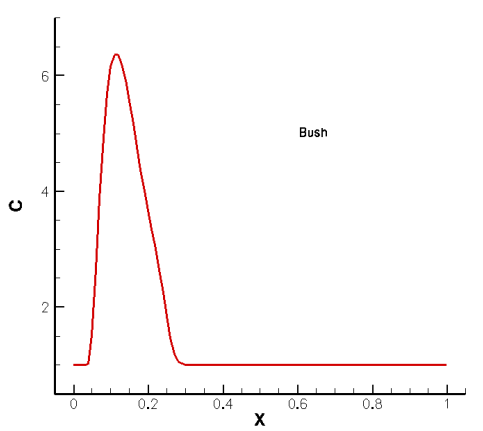

b)

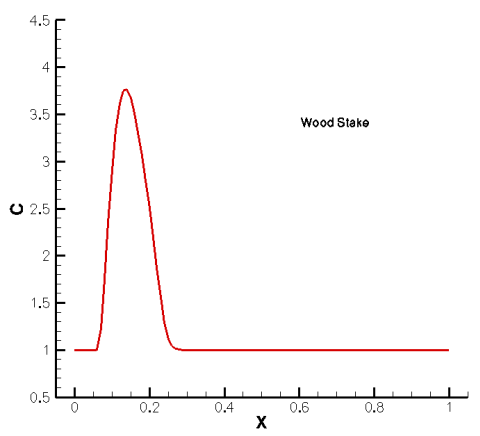

d)

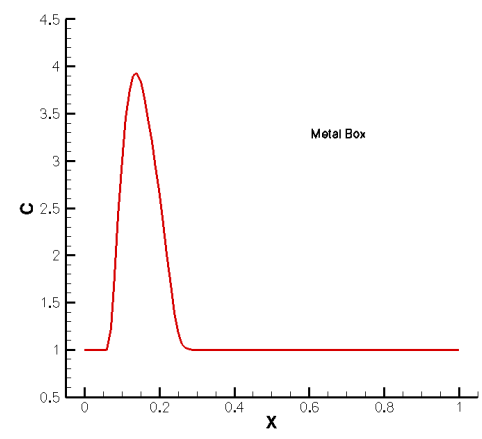

Fig. 8.1: Resulting images. a) The computed image for computationally simulated data (Test 1). Solid and dashed lines are true and computed images, respectively. Recall that we are interested in accurate imaging of target/background contrasts rather than in accurate imaging of locations of targets (subsection 7.1). The computed target/background contrast is 3.8, which is 5\% error. b) The computed image of the bush, see Figure 7.4-a). Here $\bar{R}=\bar{\varepsilon}_{r}(x)=8.5$, which is in the range of tabulated values $\varepsilon_{r} \in[3,20]$ [20]. c) Solid line is the Laplace transform of the pre-processed data for $b)$. Dashed line is the function $w(0, s)-w_{0}(0, s)$, where $w(0, s)$ is the solution of the problem (2.8), (2.9) with $\varepsilon_{r}(x):=R_{\text {comp }}(x)$ of b). d) The computed image of the wood stake, see Figure 7.4-c). Here $\bar{R}=3.8$, which is in the range of tabulated values $\varepsilon_{r} \in[2,6]$, see [45]. e) The computed image of the metal box, $\bar{R}=3.8$. 


\section{REFERENCES}

[1] Alekseenko N V, Burov V A and Rumyantseva O D 2008 Solution of the three-dimensional acoustic inverse scattering problem. The modified Novikov algorithm Acoustical Physics 54 407-419

[2] Bateman H and Erdelyi A 1954 Tables of Integral Transforms 1 (McGrawHill, New York)

[3] Beilina L, Johnson C 2001 A hybrid FEM/FDM method for an inverse scattering problem. In Numerical Mathematics and Advanced Applications - ENUMATH 2001. Springer-Verlag.

[4] Beilina L, Johnson C, A posteriori error estimation in computational inverse scattering, Mathematical Models and Methods in Applied Sciences, 15, 23-37, 2005.

[5] Beilina L and Klibanov M V 2008 A globally convergent numerical method for a coefficient inverse problem SIAM J. Sci. Comp. 31 478-509

[6] Beilina L and Klibanov M V 2010 Synthesis of global convergence and adaptivity for a hyperbolic coefficient inverse problem in 3D J. Inverse and Ill-posed Problems 18 85-132

[7] Beilina L and Klibanov M V 2010 A posteriori error estimates for the adaptivity technique for the Tikhonov functional and global convergence for a coefficient inverse problem Inverse Problems 26045012

[8] Beilina L, Klibanov M V and Kokurin M Yu 2010 Adaptivity with relaxation for ill-posed problems and global convergence for a coefficient inverse problem, J. of Mathematical Sciences 167 279-325

[9] Beilina L and Klibanov M V 2010 Reconstruction of dielectrics from experimental data via a hybrid globally convergent/adaptive algorithm Inverse Problems 26125009

[10] Beilina L, Kuzhuget A V and Klibanov M V 2011 New a posteriori error estimates for adaptivity technique and global convergence for the hyperbolic coefficient inverse problem, Journal of Mathematical Sciences 172 449-476

[11] Beilina L and Klibanov M V 2012 Approximate Global Convergence and Adaptivity for Coefficient Inverse Problems (Springer, New York), to appear in April 2012

[12] Beilina L and Klibanov M V The philosophy of the approximate global convergence for multidimensional coefficient inverse problems Complex Variables and Elliptic Equations, accepted for publication.

[13] Bikowski J, Knudsen K and Mueller J L 2011 Direct numerical reconstruction of conductivities in three dimensions using scattering transforms Inverse Problems 27015002

[14] Born M and Wolf E 1999 Principles of Optics: Electromagnetic Theory of Propagation, Interference and Diffraction of Light (Cambridge University Press, Cambridge)

[15] Bourgeois L and Darde J 2010 A duality-based method of quasi-reversibility to solve the Cauchy problem in the presence of noisy data Inverse Problems 26095016

[16] Burov V A, Morozov S A and Rumyantseva O D 2002 Reconstruction of fine-scale structure of acoustical scatterers on large-scale contrast background Acoustical Imaging 26 231-238

[17] Burov V A, Alekseenko N V and Rumyantseva O D 2009 Multifrequency generalization of the Novikov algorithm for the two-dimensional inverse scattering problem Acoustical Physics $\mathbf{5 5}$ 843-856

[18] Cao H, Klibanov M V and Pereverzev S V 2009 A Carleman estimate and the balancing principle in the quasi-reversibility method for solving the Cauchy problem for the Laplace equation Inverse Problems 2535005

[19] Chadan K and Sabatier P C 1989 Inverse Problems in Quantum Scattering Theory (Springer, New York)

[20] Chuah H T, Lee K Y and Lau T W 1995 Dielectric constants of rubber and oil palm leaf samples at X-band, IEEE Trans. on Geoscience and Remote Sensing 33 221-223

[21] DeAngelo M and J.L. Mueller J L 2010 2-D $\partial$-bar reconstructions of human chest and tank using an improved approximation to the scattering transform Physiological Measurement 31 221-232

[22] Engl H W, M. Hanke M and Neubauer A 2000 Regularization of Inverse Problems (Kluwer Academic Publishers, Boston)

[23] Friedman A 1964 Partial Differential Equations of Parabolic Type (Prentice Hall, Inc., Englewood Cliffs, N.J.)

[24] Isaacson D, Mueller J L, Newell J C and Siltanen S 2006 Imaging cardiac activity by the D-bar methods for electrical impedance tomography Physiological Measurements 27 S43-S50

[25] Kabanikhin S I and Shishlenin M A 2011 Numerical algorithm for two-dimensional inverse acoustic problem based on Gel'fand-Levitan-Krein equation J. Inverse and Ill-Posed Problems 18 979-995

[26] Kaltenbacher B, Neubauer A and Scherzer O 2008 Iterative Regularization Methods for Nonlinear Ill-Posed Problems (de Gruiter, New York)

[27] Klibanov M V, Fiddy M A, Beilina L, Pantong N and Schenk J 2010 Picosecond scale experimental verification of a globally convergent numerical method for a coefficient inverse problem Inverse Problems 26045003

[28] Klibanov M V, Bakushinsky A B and Beilina L 2011 Why a minimizer of the Tikhonov functional is closer to the exact solution than the first guess, J. Inverse and Ill-Posed Problems 19 83-105

[29] Klibanov M V, Su J, Pantong N, Shan H and Liu H 2010 A globally convergent numerical method for an inverse elliptic problem of optical tomography Applicable Analysis 89 861-891

[30] Klibanov M V and Timonov A 2004 Carleman Estimates for Coefficient Inverse Problems and Numerical Applications (VSP, Utrecht, The Netherlands)

[31] Kuzhuget A V and M.V. Klibanov M V 2010 Global convergence for a 1-D inverse problem with application to imaging of land mines Applicable Analysis 89 125-157

[32] Kuzhuget A V, Pantong N and Klibanov M V 2011 A globally convergent numerical method for a Coefficient Inverse Problem with backscattering data Methods and Applications of Analysis 18 47-68

[33] Ladyzhenskaya O A, Solonnikov V A and Uralceva N N 1968 Linear and Quasilinear Equations of Parabolic Type 
(AMS, Providence, R.I.)

[34] Lattes R and Lions J-L 1969 The Method of Quasireversibility: Applications to Partial Differential Equations (Elsevier, New York)

[35] Lavrentiev M M, Romanov V G and Shishatskii S P 1986 Ill-Posed Problems of Mathematical Physics and Analysis (AMS, Providence, R.I.)

[36] Levitan B M 1987 Inverse Sturm-Liouville Problems (VSP, Utrecht, The Netherlands)

[37] Nguyen N, Wong D, Ressler M, Koenig F,Stanton B, Smith G, Sichina J and Kappra K 2007 Obstacle avolidance and concealed target detection using the Army Research Lab ultra-wideband synchronous impulse Reconstruction (UWB SIRE) forward imaging radar Proc. SPIE $655365530 \mathrm{H}$ (1)-65530H (8)

[38] Novikov R G 1988 Multidimensional inverse spectral problem for the equation $-\triangle \psi+(v(x)-E u(x)) \psi=0$. Funkc. Anal. Priloz. 2284 11-22

[39] Novikov R G 1992 The inverse scattering problem on a fixed energy level for the two-dimensional Schrödinger operator J. Funct.Anal. 103 409-463

[40] Novikov R G 2005 The $\partial$-bar approach to approximate inverse scattering at fixed energy in three dimensions, Int. Math. Res. Pap. 6 287-349

[41] Novikov R G 2009 An effectivization of the global reconstruction in the Gel'fand-Calderon inverse problem in three dimensions Contemporary Mathematics 494 161-184

[42] Pantong N, Su J, Shan H, Klibanov M V and Liu H 2009 Globally accelerated reconstruction algorithm for diffusion tomography with continuous-wave source in an arbitrary convex shape domain J. Optical Society of America A 26 456-472

[43] Shan H, Klibanov M V, Su J, Pantong N and Liu H 2008 A globally accelerated numerical method for optical tomography with continuous wave source J. Inverse and Ill-Posed Problems 16 765-792

[44] Su J, Klibanov M V, Liu Y, Lin Z, Pantong N and Liu H 2011 An inverse elliptic problem of medical optics with experimental data, preprint, available on-line at http://www.ma.utexas.edu/mp_arc/.

[45] Tables of dielectric constants at http://www.asiinstr.com/technical/Dielectric0Constants.htm.

[46] Tables of dielectric constants at http://www.krohne.com/Dielectric_Constants.6840.0.html.

[47] Tikhonov A N, Goncharsky A V, Stepanov V V and Yagola A G 1995 Numerical Methods for the Solution of Ill-Posed Problems (Kluwer, London)

[48] Xin J, Beilina L and Klibanov M V 2010 Globally convergent numerical methods for coefficient inverse problems for imaging inhomogeneities Computing in Science and Engineering 12 No. 5, 64-77 